\title{
An Evolutionary Variational Inequality Formulation of Supply Chain Networks with Time-Varying Demands
}

\author{
Anna Nagurney and Zugang Liu \\ Department of Finance and Operations Management \\ Isenberg School of Management \\ University of Massachusetts \\ Amherst, Massachusetts 01003 \\ December 2005; updated June 2006 and February 2007 \\ Appears in: Network Science, Nonlinear Science and Dynamic Game Theory \\ Applied to the Study of Infrastructure Systems (2007), pp. 267-302 \\ Terry L. Friesz, Editor, Springer
}

\begin{abstract}
This paper first develops a multitiered supply chain network equilibrium model with fixed demands and proves that the governing equilibrium conditions satisfy a finitedimensional variational inequality. The paper then establishes that the static supply chain network model with its governing equilibrium conditions can be reformulated as a transportation network equilibrium model over an appropriately constructed abstract network or supernetwork. This identification provides a new interpretation of equilibrium in supply chain networks with fixed demands in terms of path flows. The equivalence is then further exploited to construct a dynamic supply chain network model with time-varying demands (and flows) using an evolutionary (time-dependent) variational inequality formulation. Recent theoretical results in the unification of projected dynamical systems and evolutionary variational inequalities are presented and then applied to formulate dynamic numerical supply chain network examples and to compute the curves of equilibria. An example with step-wise time-dependent demand is also given for illustration purposes.
\end{abstract}




\section{Acknowledgments}

This research was supported by NSF Grant No. IIS - 002647. The first author also acknowledges support from the Radcliffe Institute for Advanced Study at Harvard University under its 2005 - 2006 Felowship Program. The support provided is gratefully acknowledged.

The authors would also like to thank Terry L. Friesz for organizing and hosting the NSFsponsored workshop at Pennsylvania State University, May 9-11, 2005, on Network Science, Nonlinear Science and Infrastructure Systems at which an earlier version of this paper was presented. Finally, thanks are extended to the participants for stimulating discussions at the workshop. 


\section{Introduction}

Transportation science has pushed the frontiers in the development and application of rigorous methodologies for the modeling, analysis, and solution of complex network-based problems in which humans interact with critical infrastructure as well as with available technologies. Seminal contributions have been made by numerous authors. Beckmann, McGuire, and Winsten (1956) first rigorously mathematically formulated the traffic assignment or transportation network equilibrium problem and showed that the equilibrium conditions in which no user has any incentive to alter his route of travel coincided with the Kuhn-Tucker conditions of an appropriately constructed optimization problem. Dafermos and Sparrow (1969), subsequently, coined the terms "user-optimization" and "system-optimization" to distinguish between solutions corresponding to, respectively, Wardrop's (1952) first and second principles of travel behavior and also provided algorithms that exploited the network structure. Dafermos (1980) identified the Smith (1979) formulation of transportation network equilibrium as a finite-dimensional variational inequality, an identification that revolutionized the modeling of a spectrum of network equilibrium problems and applications in different disciplines. For an overview of the impact of the Beckmann, McGuire, and Winsten (1956) book, see the paper by Boyce, Mahmassani, and Nagurney (2005). For an overview of finite-dimensional variational inequalities and network-based applications, see the book by Nagurney (1999) and the references therein.

Transportation science has also spearheaded the development of mathematical frameworks to capture disequilibrium behavior associated with the dynamics of users engaged in selecting their routes of travel between origins and destinations. For example, projected dynamical systems theory (cf. Dupuis and Nagurney (1993) and Nagurney and Zhang (1996) and the references therein) was developed, in part, in order to model the behavior of travelers prior to the achievement of an equilibrium state as formulated by a variational inequality problem. The book by Ran and Boyce (1996) contains an overview of dynamic transportation network models and algorithms, along with references to the literature to that date. Formulations of dynamic models of multilayer networks can be found in Nagurney and Dong (2002) and in Zhang, Peeta, and Friesz (2005).

Recently, Cojocaru, Daniele, and Nagurney (2005) built the basis for the merging of pro- 
jected dynamical systems and that of evolutionary (time-dependent) variational inequalities (which are infinite-dimensional). These two theories had been developed and advanced in parallel, in order to further develop the theoretical analysis and computation of solutions to problems, often, network-based, in which dynamics plays a central role. Cojocaru, Daniele, and Nagurney (2006) demonstrated that the merger of these two theories allows for the modeling of problems that present two theoretically distinct timeframes. They provided the formulation of the associated double-dynamics theory, discussed the question of uniqueness of such curves of equilibria, and also provided conditions for stability properties of such curves in a given neighborhood.

In this paper, we focus on the interplay of transportation network equilibrium models and supply chain network problems and we demonstrate how theory derived from the former class of problems can be used to provide entirely new interpretations of the latter as well as to inform a new kind of time-dependent modeling framework, which is motivated by the unification of projected dynamical systems theory and evolutionary variational inequalities. This paper is organized as follows. In Section 2, we present the multitiered supply chain network equilibrium model with fixed demands, which is motivated by the supply chain network equilibrium model proposed by Nagurney, Dong, and Zhang (2002). In Section 3, we then recall the fixed demand transportation network equilibrium model of Dafermos (1980) and Smith (1979), along with the path-based and link-based variational inequality formulations, which are finite-dimensional. In Section 4, we establish that the supply chain network equilibrium model of Section 2 can be reformulated as a transportation network equilibrium model as described in Section 3, over an appropriately constructed abstract network or supernetwork (cf. Nagurney and Dong (2002) and the references therein). A similar equivalence was made by Nagurney (2006) for the case of elastic demands.

In Section 4, we utilize the recently developed unification of the theories of projected dynamical systems and evolutionary variational inequalities to construct a dynamic supply chain network model with time-dependent demands, which is viewed as a dynamic transportation network problem. We also provide some theoretical results. In Section 5, we discuss the computation of curves of equilibria and we illustrate the modeling framework through several numerical dynamic supply chain numerical examples, including one with a step-wise demand function. 


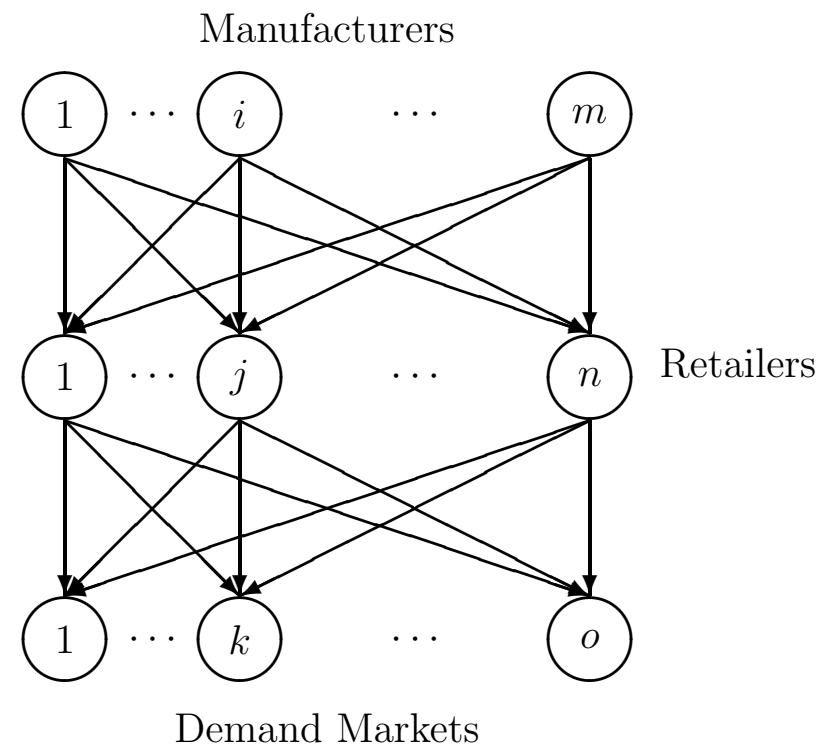

Figure 1: The Network Structure of the Supply Chain

\section{The Supply Chain Network Model with Fixed Demands}

In this Section, we develop a fixed demand version of the supply chain network model proposed in Nagurney, Dong, and Zhang (2002). The model consists of $m$ manufacturers, $n$ retailers, and $o$ demand markets, as depicted in Figure 1. We denote a typical manufacturer by $i$, a typical retailer by $j$, and a typical demand market by $k$. The links in the supply chain network represent transportation/transaction links. The majority of the needed notation is shown in Table 1. The equilibrium solution is denoted by "**". All vectors are assumed to be column vectors, except where noted.

The top-tiered nodes in Figure 1 represent the manufacturers, who produce a homogeneous product and sell to the retailers in the second tier. The consumers at the demand markets are represented by the nodes in the bottom tier of the supply chain network and they acquire the product from the retailers. 
Table 1: Notation for the Supply Chain Network Equilibrium Model

\begin{tabular}{|l|l|}
\hline Notation & Definition \\
\hline$q$ & $\begin{array}{l}\text { vector of the manufacturers' production outputs with components: } \\
q_{1}, \ldots, q_{m} \\
m n \text {-dimensional vector of product flows transacted/shipped between } \\
\text { manufacturers and retailers with component } i j: q_{i j} \\
\text { no-dimensional vector of product flows transacted/shipped between } \\
\text { retailers and the demand markets with component } j k: q_{j k} \\
n \text {-dimensional vector of shadow prices associated with the retailers } \\
\text { with component } j: \gamma_{j}\end{array}$ \\
$\gamma$ & $\begin{array}{l}o \text {-dimensional vector of market demand with component } k: d_{k} \\
\text { production cost of manufacturer } i \text { with marginal production cost } \\
\text { with respect to } q_{i}: \frac{\partial f_{i}}{\partial q_{i}} \text { and the marginal production cost with } \\
\text { respect to } q_{i j}: \frac{\partial f_{i}\left(Q^{1}\right)}{\partial q_{i j}} \\
\text { transaction cost between manufacturer } i \text { and retailer } j \text { with marginal } \\
\text { transaction cost: } \frac{\partial c_{i j}\left(q_{i j}\right)}{\partial q_{i j}} \\
f_{i}(q) \equiv f_{i}\left(Q^{2}\right. \\
c_{i j}\left(q_{i j}\right)\end{array}$ \\
$s$ & $\begin{array}{l}s_{1}, \ldots, s_{n} \\
\text { handling } \operatorname{cost} \text { of retailer } j \text { with marginal handling cost with respect } \\
\text { to } s_{j}: \frac{\partial c_{j}}{\partial s_{j}} \text { and with the marginal handling cost with respect to } q_{i j}: \\
\frac{\partial c_{j}\left(Q^{1}\right)}{\partial q_{i j}} \\
\text { unit transaction cost between retailer } j \text { and demand market } k\end{array}$ \\
$c_{j}(s) \equiv c_{j}\left(Q^{2}\right.$
\end{tabular}


We first describe the behavior of the manufacturers and the retailers. We then discuss the behavior of the consumers at the demand markets. Finally, we state the equilibrium conditions for the supply chain network and provide the finite-dimensional variational inequality governing the equilibrium.

\subsection{The Behavior of the Manufacturers and their Optimality Conditions}

Let $\rho_{1 i j}^{*}$ denote the price charged for the product by manufacturer $i$ in transacting with retailer $j$. The price $\rho_{1 i j}^{*}$ is an endogenous variable and will be determined once the entire supply chain network equilibrium model is solved. We assume that the quantity produced by manufacturer $i$ must satisfy the following conservation of flow equation:

$$
q_{i}=\sum_{j=1}^{n} q_{i j}
$$

which states that the quantity of the product produced by manufacturer $i$ is exactly equal to the sum of the quantities transacted/shipped between a manufacturer and the retailers. The production cost function $f_{i}$ for each manufacturer $i ; i=1, \ldots, m$, as delineated in Table 1 , can, in view of (1), be reexpressed as a function of the flows $Q^{1}$.

Hence, assuming that the manufacturers are profit-maximizers, we can express the optimization problem faced by manufacturer $i$ as:

$$
\text { Maximize } \sum_{j=1}^{n} \rho_{1 i j}^{*} q_{i j}-f_{i}\left(Q^{1}\right)-\sum_{j=1}^{n} c_{i j}\left(q_{i j}\right)
$$

subject to: $q_{i j} \geq 0$, for all $j ; j=1, \ldots, n$.

The first term in (2) represents the revenue and the subsequent two terms the production cost and the transaction costs, respectively, for manufacturer $i$.

We assume that the manufacturers compete in a noncooperative manner in the sense of Cournot (1838) and Nash $(1950,1951)$, and the production cost functions and the transaction cost functions for each manufacturer are continuously differentiable and convex. The optimality conditions for all manufacturers $i ; i=1, \ldots, m$, simultaneously, can then be expressed as the following variational inequality (cf. Nagurney, Dong and Zhang (2002), 
Bazaraa, Sherali, and Shetty (1993), Gabay and Moulin (1980); see also Dafermos and Nagurney (1987) and Nagurney (1999)): determine $Q^{1 *} \in R_{+}^{m n}$ satisfying:

$$
\sum_{i=1}^{m} \sum_{j=1}^{n}\left[\frac{\partial f_{i}\left(Q^{1 *}\right)}{\partial q_{i j}}+\frac{\partial c_{i j}\left(q_{i j}^{*}\right)}{\partial q_{i j}}-\rho_{1 i j}^{*}\right] \times\left[q_{i j}-q_{i j}^{*}\right] \geq 0, \quad \forall Q^{1} \in R_{+}^{m n} .
$$

\subsection{The Behavior of the Retailers and their Optimality Conditions}

The retailers, in turn, purchase the product from the manufacturers and transact with the consumers at the demand markets. Thus, a retailer is involved in transactions both with the manufacturers as well as with the demand markets.

Let $\rho_{2 j}^{*}$ denote the price charged by the retailer $j$ for the product. This price will be determined endogenously after the the model is solved. We assume that the retailers are also profit-maximizers and, hence, the optimization problem faced by a retailer $j$ is given by:

$$
\operatorname{Maximize} \quad \sum_{k=1}^{o} \rho_{2 j}^{*} q_{j k}-c_{j}\left(Q^{1}\right)-\sum_{i=1}^{m} \rho_{1 i j}^{*} q_{i j}
$$

subject to:

$$
\sum_{k=1}^{o} q_{j k} \leq \sum_{i=1}^{m} q_{i j}
$$

and the nonnegativity constraints: $q_{i j} \geq 0$, and $q_{j k} \geq 0$, for all $i ; i=1, \ldots, m$, and $k$; $k=1, \ldots, o$.

The first term in the objective function (4) represents the revenue whereas the second and third terms represent, respectively, the handling cost and the payout to the manufacturers. Constraint (5) expresses that the total quantity of the product transacted with the demand markets by a retailer cannot exceed the total amount that the retailer has obtained from the manufacturers.

We assume that the retailers also compete in a noncooperative manner and that the handling cost for each retailer is continuously differentiable and convex. Then the optimality conditions for all the retailers simultaneously can be expressed as the variational inequality: 
determine $\left(Q^{1 *}, Q^{2 *}, \gamma^{*}\right) \in R_{+}^{m n+n o+n}$ satisfying:

$$
\begin{gathered}
\sum_{i=1}^{m} \sum_{j=1}^{n}\left[\frac{\partial c_{j}\left(Q^{1 *}\right)}{\partial q_{i j}}+\rho_{1 i j}^{*}-\gamma_{j}^{*}\right] \times\left[q_{i j}-q_{i j}^{*}\right]+\sum_{j=1}^{n} \sum_{k=1}^{o}\left[-\rho_{2 j}^{*}+\gamma_{j}^{*}\right] \times\left[q_{j k}-q_{j k}^{*}\right] \\
+\sum_{j=1}^{n}\left[\sum_{i=1}^{m} q_{i j}^{*}-\sum_{k=1}^{o} q_{j k}^{*}\right] \times\left[\gamma_{j}-\gamma_{j}^{*}\right] \geq 0, \quad \forall\left(Q^{1}, Q^{2}, \gamma\right) \in R_{+}^{m n+n o+n} .
\end{gathered}
$$

As noted in Table 1, the term $\gamma_{j}$ is the Lagrange multiplier/shadow price associated with constraint (5) for retailer $j$ and $\gamma$ is the $n$-dimensional vector of all the shadow prices.

\subsection{The Consumers at the Demand Markets and the Equilibrium Conditions}

We now discuss the behavior of the consumers at the demand markets. The consumers take into account the prices charged by the retailers and the unit transaction costs incurred to obtain the product in making their consumption decisions. In the static model, we assume that the demand for the product at each demand market is fixed and known (later in this paper, we will develop the dynamic model in which we allow the demand to be time-varying). Hence, the following conservation of flow equations must hold:

$$
d_{k}=\sum_{j=1}^{n} q_{j k}, \quad k=1, \ldots, o,
$$

where $d_{k}$ is fixed for each demand market $k$.

We assume that the unit transaction cost functions $c_{j k}$ are continuous functions for $j$; $j=1, \ldots, n$ and $k ; k=1, \ldots, o$, and are of the form given in Table 1 .

The equilibrium conditions for consumers at demand market $k$ then take the form: for each retailer $j ; j=1, \ldots, n$ :

$$
\rho_{2 j}^{*}+c_{j k}\left(Q^{2 *}\right)\left\{\begin{array}{lll}
=\rho_{3 k}^{*}, & \text { if } & q_{j k}^{*}>0 \\
\geq \rho_{3 k}^{*}, & \text { if } & q_{j k}^{*}=0
\end{array}\right.
$$

Conditions (8) state that, in equilibrium, if the consumers at demand market $k$ purchase the product from retailer $j$, then the price the consumers pay is exactly equal to the price charged by the retailer plus the unit transaction cost. However, if the sum of the 
price charged by the retailer and the unit transaction cost exceeds the price that the consumers are willing to pay at the demand market, there will be no transaction between this retailer/demand market pair. In equilibrium, condition (8) must hold simultaneously for all demand markets. We can express these equilibrium conditions as the following variational inequality: determine $Q^{2 *} \in \mathcal{K}^{1}$, such that

$$
\sum_{j=1}^{n} \sum_{k=1}^{o}\left[\rho_{2 j}^{*}+c_{j k}\left(Q^{2 *}\right)\right] \times\left[q_{j k}-q_{j k}^{*}\right] \geq 0, \quad \forall Q^{2} \in \mathcal{K}^{1},
$$

where $\mathcal{K}^{1} \equiv\left\{Q^{2} \mid Q^{2} \in R_{+}^{n o}\right.$ and (7) holds $\}$.

In Nagurney, Dong, and Zhang (2002), it was assumed that the demand functions associated with the demand markets were elastic and depended upon the prices of the product at the demand markets. Nagurney (2006), subsequently, proved that the elastic demand supply chain network equilibrium model could be reformulated as an elastic demand transportation network equilibrium model (cf. Dafermos and Nagurney (1984); see also Fisk and Boyce (1983)) over an appropriately constructed supernetwork. That paper, however, did not introduce any dynamics.

\subsection{The Equilibrium Conditions of the Supply Chain}

In equilibrium, the optimality conditions of all the manufacturers, the optimality conditions of all the retailers, and the equilibrium conditions for all the demand markets must be simultaneously satisfied so that no decision-maker has any incentive to alter his transactions. We now formally state the equilibrium conditions for the entire supply chain network as follows.

\section{Definition 1: Supply Chain Network Equilibrium (Fixed Demands)}

The equilibrium state of the supply chain network with fixed demands is one where the flows of the product between the tiers of the decision-makers coincide and the flows and prices satisfy the sum of conditions (3), (6), and (9).

We now state and prove: 


\section{Theorem 1: Variational Inequality Formulation of the Supply Chain Network Equilibrium}

The equilibrium conditions governing the supply chain network according to Definition 1 coincide with the solution of the (finite-dimensional) variational inequality given by: determine $\left(Q^{1 *}, Q^{2 *}, \gamma^{*}\right) \in \mathcal{K}^{2}$ satisfying:

$$
\begin{gathered}
\sum_{i=1}^{m} \sum_{j=1}^{n}\left[\frac{\partial f_{i}\left(Q^{1^{*}}\right)}{\partial q_{i j}}+\frac{\partial c_{i j}\left(q_{i j}^{*}\right)}{\partial q_{i j}}+\frac{\partial c_{j}\left(Q^{1^{*}}\right)}{\partial q_{i j}}-\gamma_{j}^{*}\right] \times\left[q_{i j}-q_{i j}^{*}\right] \\
+\sum_{j=1}^{n} \sum_{k=1}^{o}\left[c_{j k}\left(Q^{2^{*}}\right)+\gamma_{j}^{*}\right] \times\left[q_{j k}-q_{j k}^{*}\right]+\sum_{j=1}^{n}\left[\sum_{i=1}^{m} q_{i j}^{*}-\sum_{k=1}^{o} q_{j k}^{*}\right] \times\left[\gamma_{j}-\gamma_{j}^{*}\right] \geq 0, \\
\forall\left(Q^{1}, Q^{2}, \gamma\right) \in \mathcal{K}^{2} .
\end{gathered}
$$

where $\mathcal{K}^{2} \equiv\left\{\left(Q^{1}, Q^{2}, \gamma\right) \mid\left(Q^{1}, Q^{2}, \gamma\right) \in R_{+}^{m n+n o+n}\right.$ and $(7)$ holds $\}$.

Proof: We first demonstrate that an equilibrium pattern according to Definition 1 satisfies the variational inequality (10). We sum up inequalities (3), (6), and (9), and, after algebraic simplifications, obtain (10).

We now prove the converse, that is, a solution to variational inequality (10) satisfies the sum of conditions (3), (6), and (9), and is, therefore, a supply chain network equilibrium pattern according to Definition 1.

First, we add the term $\rho_{1 i j}^{*}-\rho_{1 i j}^{*}$ to the first term in the first summand expression in (10). Then, we add the term $\rho_{2 j}^{*}-\rho_{2 j}^{*}$ to the first term in the second summand expression in (10). Because these terms are all equal to zero, they do not change (10) and we obtain the following inequality:

$$
\begin{gathered}
\sum_{i=1}^{m} \sum_{j=1}^{n}\left[\frac{\partial f_{i}\left(Q^{1 *}\right)}{\partial q_{i j}}+\frac{\partial c_{i j}\left(q_{i j}^{*}\right)}{\partial q_{i j}}+\frac{\partial c_{j}\left(Q^{1 *}\right)}{\partial q_{i j}}-\gamma_{j}^{*}+\rho_{1 i j}^{*}-\rho_{1 i j}^{*}\right] \times\left[q_{i j}-q_{i j}^{*}\right] \\
+\sum_{j=1}^{n} \sum_{k=1}^{o}\left[c_{j k}\left(Q^{2 *}\right)+\gamma_{j}^{*}+\rho_{2 j}^{*}-\rho_{2 j}^{*}\right] \times\left[q_{j k}-q_{j k}^{*}\right] \\
+\sum_{j=1}^{n}\left[\sum_{i=1}^{m} q_{i j}^{*}-\sum_{k=1}^{o} q_{j k}^{*}\right] \times\left[\gamma_{j}-\gamma_{j}^{*}\right] \geq 0, \quad \forall\left(Q^{1}, Q^{2}, \gamma\right) \in \mathcal{K}^{2},
\end{gathered}
$$


which can be rewritten as:

$$
\begin{gathered}
\sum_{i=1}^{m} \sum_{j=1}^{n}\left[\frac{\partial f_{i}\left(Q^{1 *}\right)}{\partial q_{i j}}+\frac{\partial c_{i j}\left(q_{i j}^{*}\right)}{\partial q_{i j}}-\rho_{1 i j}^{*}\right] \times\left[q_{i j}-q_{i j}^{*}\right] \\
\sum_{i=1}^{m} \sum_{j=1}^{n}\left[\frac{\partial c_{j}\left(Q^{1 *}\right)}{\partial q_{i j}}+\rho_{1 i j}^{*}-\gamma_{j}^{*}\right] \times\left[q_{i j}-q_{i j}^{*}\right]+\sum_{j=1}^{n} \sum_{k=1}^{o}\left[-\rho_{2 j}^{*}+\gamma_{j}^{*}\right] \times\left[q_{j k}-q_{j k}^{*}\right] \\
+\sum_{j=1}^{n}\left[\sum_{i=1}^{m} q_{i j}^{*}-\sum_{k=1}^{o} q_{j k}^{*}\right] \times\left[\gamma_{j}-\gamma_{j}^{*}\right] \\
+\sum_{j=1}^{n} \sum_{k=1}^{o}\left[\rho_{2 j}^{*}+c_{j k}\left(Q^{2 *}\right)\right] \times\left[q_{j k}-q_{j k}^{*}\right] \geq 0, \quad \forall\left(Q^{1}, Q^{2}, \gamma\right) \in \mathcal{K}^{2} .
\end{gathered}
$$

Clearly, (12) is equal to the sum of the optimality conditions (3) and (6), and the equilibrium conditions (9) and is, hence, a variational inequality governing the supply chain network equilibrium according to Definition 1.

The variational inequality (10) is different from the variational inequality formulation of elastic demand supply chain network equilibrium problems derived by Nagurney, Dong, and Zhang (2002), as expected, since the feasible set is different and we do not have demand functions, but, rather, we now assume fixed demands.

At the end of Section 4, we describe how to recover the nodal prices in the supply chain network with fixed demands consisting of the top tier prices: $\rho_{1 i j}^{*}$; for $i=1, \ldots, m$; $j=1, \ldots, n$; the middle tier prices: $\rho_{2 j}^{*} ; j=1, \ldots, n$, and the demand market prices: $\rho_{3 k}^{*}$; $k=1, \ldots, o$. The nodal prices of the supply chain network guarantee that the optimality conditions (3), (6), and the equilibrium conditions (8) hold separately at the solution of the variational inequality (10).

The following corollary establishes that, in equilibrium, the supply chain structure is as in Figure 1. Of course, links that have equilibrium flows of zero can, in effect, be eliminated from the supply chain network. This corollary is also useful in establishing the equivalence of the supply chain network equilibrium problem with fixed demands with the transportation network equilibrium problem with fixed demands over an appropriately constructed abstract network or supernetwork, as we demonstrate in the next Section. 


\section{Corollary 1}

The market for the product clears for each retailer in the supply chain network equilibrium, that is, $\sum_{i=1}^{m} q_{i j}^{*}=\sum_{k=1}^{o} q_{j k}^{*}$ for $j=1, \ldots, n$.

Proof: Clearly from (10), we know that, if $\gamma_{j}^{*}>0$, then $\sum_{i=1}^{m} q_{i j}^{*}=\sum_{k=1}^{o} q_{j k}^{*}$ holds. Now we consider the case where $\gamma_{j}^{*}=0$ for some retailer $j$. Let us examine the first terms in inequality (10). Since we have assumed that the production cost functions, the transaction cost functions, and the handling cost functions are convex, it is not unreasonable to further assume that either the marginal production cost or the marginal transaction cost or the marginal handling cost for each manufacturer/retailer pair is strictly positive at equilibrium. Then we know that $\frac{\partial f_{i}\left(Q^{1 *}\right)}{\partial q_{i j}}+\frac{\partial c_{i j}\left(q_{i j}^{*}\right)}{\partial q_{i j}}+\frac{\partial c_{j}\left(Q^{1 *}\right)}{\partial q_{i j}}>0$, which implies that $q_{i j}^{*}=0$, for all $i$, for that $j$. It follows then from the third term in (10), that $\sum_{k=1}^{o} q_{j k}^{*}=0$. Hence, we have that $\sum_{k=1}^{o} q_{j k}^{*}=0=\sum_{i=1}^{m} q_{i j}^{*}$ for any $j$ such that $\gamma_{j}^{*}=0$. Therefore, we conclude that the market clears for each retailer in the supply chain equilibrium.

Since we are interested in the determination of the equilibrium flows and prices, we can transform constraint (5) into:

$$
\sum_{k=1}^{o} q_{j k}=\sum_{i=1}^{m} q_{i j}, \quad j=1, \ldots, n .
$$

Now we can define the feasible set as $\mathcal{K}^{3} \equiv\left\{\left(Q^{1}, Q^{1}\right) \in R_{+}^{m n+n o}\right.$ such that (13) holds $\}$.

In addition, for notational convenience, we let

$$
s_{j} \equiv \sum_{k=1}^{o} q_{j k}, \quad j=1, \ldots, n .
$$

The following results then follow immediately:

\section{Corollary 2}

A solution $\left(Q^{1 *}, Q^{2 *}\right) \in \mathcal{K}^{3}$ to the variational inequality problem:

$$
\sum_{i=1}^{m} \sum_{j=1}^{n}\left[\frac{\partial f_{i}\left(Q^{1 *}\right)}{\partial q_{i j}}+\frac{\partial c_{i j}\left(q_{i j}^{*}\right)}{\partial q_{i j}}+\frac{\partial c_{j}\left(Q^{1 *}\right)}{\partial q_{i j}}\right] \times\left[q_{i j}-q_{i j}^{*}\right]
$$




$$
+\sum_{j=1}^{n} \sum_{k=1}^{o}\left[c_{j k}\left(Q^{2 *}\right)\right] \times\left[q_{j k}-q_{j k}^{*}\right] \geq 0, \quad \forall\left(Q^{1}, Q^{2}\right) \in \mathcal{K}^{3}
$$

equivalently, a solution $\left(q^{*}, Q^{1 *}, s^{*}, Q^{2 *}\right) \in \mathcal{K}^{4}$ to:

$$
\begin{gathered}
\sum_{i=1}^{m}\left[\frac{\partial f_{i}\left(q^{*}\right)}{\partial q_{i}}\right] \times\left[q_{i}-q_{i}^{*}\right]+\sum_{i=1}^{m} \sum_{j=1}^{n}\left[\frac{\partial c_{i j}\left(q_{i j}^{*}\right)}{\partial q_{i j}}\right] \times\left[q_{i j}-q_{i j}^{*}\right]+\sum_{j=1}^{n}\left[\frac{\partial c_{j}\left(s^{*}\right)}{\partial s_{j}}\right] \times\left[s_{j}-s_{j}^{*}\right] \\
+\sum_{j=1}^{n} \sum_{k=1}^{o}\left[c_{j k}\left(Q^{2 *}\right)\right] \times\left[q_{j k}-q_{j k}^{*}\right] \geq 0, \quad \forall\left(q, Q^{1}, s, Q^{2}\right) \in \mathcal{K}^{4}
\end{gathered}
$$

where $\mathcal{K}^{4} \equiv\left\{\left(q, Q^{1}, s, Q^{2}\right) \mid\left(q, Q^{1}, s, Q^{2}\right) \in R_{+}^{m+m n+n+n o}\right.$ and $(1),(7),(13)$, and $(14)$, hold $\}$, also satisfies variational inequality (10).

Proof: We prove Corollary 2 by contradiction. In particular, we demonstrate that if (10) is not true, then (15) does not hold. We assume that for some $\left(Q^{1}, Q^{2}\right) \in \mathcal{K}^{3}$ with $\gamma \in R_{+}^{n}$ that the left-hand side of (10) is less than or equal to zero, which implies that:

$$
\begin{gathered}
\sum_{i=1}^{m} \sum_{j=1}^{n}\left[\frac{\partial f_{i}\left(Q^{1 *}\right)}{\partial q_{i j}}+\frac{\partial c_{i j}\left(q_{i j}^{*}\right)}{\partial q_{i j}}+\frac{\partial c_{j}\left(Q^{1 *}\right)}{\partial q_{i j}}\right] \times\left[q_{i j}-q_{i j}^{*}\right] \\
+\sum_{j=1}^{n} \sum_{k=1}^{o} c_{j k}\left(Q^{2 *}\right) \times\left[q_{j k}-q_{j k}^{*}\right] \\
\leq \sum_{i=1}^{m} \sum_{j=1}^{n} \gamma_{j}^{*}\left[q_{i j}-q_{i j}^{*}\right]-\sum_{j=1}^{n} \sum_{k=1}^{o} \gamma_{j}^{*}\left[q_{j k}-q_{j k}^{*}\right]-\sum_{j=1}^{n}\left[\sum_{i=1}^{m} q_{i j}^{*}-\sum_{k=1}^{o} q_{j k}^{*}\right] \times\left[\gamma_{j}-\gamma_{j}^{*}\right] .
\end{gathered}
$$

But, after algebraic simplification and the use of Corollary 1, the right-hand side of (17) is reduced to zero. Hence, (15) cannot hold, and the conclusion follows.

We can obtain variational inequality (16) from variational inequality (15) through simple algebraic relationships and the use of (1), (13), and (14). 


\section{The Transportation Network Equilibrium Model with Fixed Demands}

In this Section, we review the transportation network equilibrium model with fixed demands, due to Smith (1979) and Dafermos (1980).

Consider a network $\mathcal{G}$ with the set of links $L$ with $K$ elements, the set of paths $P$ with $Q$ elements, and the set of origin/destination (O/D) pairs $W$ with $Z$ elements. We denote the set of paths connecting $\mathrm{O} / \mathrm{D}$ pair $w$ by $P_{w}$; the links by $a, b$, etc; the paths by $p, q$, etc., and the $\mathrm{O} / \mathrm{D}$ pairs by $w_{1}, w_{2}$, etc.

The flow on path $p$ is denoted by $x_{p}$ and the flow on link $a$ by $f_{a}$. The travel cost experienced by a user on a path $p$ is denoted by $C_{p}$ and the travel cost incurred on a link $a$ by $c_{a}$. We assume that the user link cost functions are continuous. We also denote the travel demand associated with traveling between $\mathrm{O} / \mathrm{D}$ pair $w$ by $d_{w}$ and the travel disutility by $\lambda_{w}$, where $d_{w}$ is assumed to be fixed and known for all $w$.

Hence, the following conservation of flow equations must hold:

$$
d_{w}=\sum_{p \in P_{w}} x_{p}, \quad \forall w,
$$

that is, the travel demand associated with an $\mathrm{O} / \mathrm{D}$ pair must be equal to the sum of the flows on the paths that connect that $\mathrm{O} / \mathrm{D}$ pair.

The following conservation of flow equations relate the link flows to the path flows:

$$
f_{a}=\sum_{p \in P} x_{p} \delta_{a p}, \quad \forall a \in L,
$$

where $\delta_{a p}=1$, if path $p$ contains link $a$, and $\delta_{a p}=0$, otherwise. Hence, the flow on a link is equal to the sum of the flows of paths that contain that link.

The user cost on a path is equal to the sum of user costs on links the path consists of, which can be represented by the following:

$$
C_{p}=\sum_{a \in L} c_{a} \delta_{a p}, \quad \forall p \in P,
$$

For the sake of generality, we allow the user cost on a link to depend upon the entire 
vector of link flows, denoted by $f$, so that

$$
c_{a}=c_{a}(f), \quad \forall a \in L .
$$

As established by Smith (1979) and Dafermos (1980), a path flow pattern $x^{*} \in \mathcal{K}^{5}$, where $\mathcal{K}^{5} \equiv\left\{x \mid x \in R_{+}^{Q}\right.$ and (18) holds $\}$ is said to be a transportation network equilibrium (according to Wardrop's first principle; see Wardrop (1952) and Beckmann, McGuire, and Winsten (1956)), if, once established, no user has any incentive to alter his travel decisions. The state can be expressed by the following equilibrium conditions which must hold for every $\mathrm{O} / \mathrm{D}$ pair $w \in W$ and every path $p \in P_{w}$ :

$$
C_{p}\left(x^{*}\right)-\lambda_{w}^{*}\left\{\begin{array}{lll}
=0, & \text { if } & x_{p}^{*}>0, \\
\geq 0, & \text { if } & x_{p}^{*}=0 .
\end{array}\right.
$$

Conditions (22) express that the user costs of all utilized paths joining an O/D pair are equal and minimal. As described in Dafermos (1980) and Smith (1979) the transportation network equilibrium pattern according to conditions (22) coincides to the following finitedimensional variational inequality in path flows: determine $x^{*} \in \mathcal{K}^{5}$ such that

$$
\sum_{w \in W} \sum_{p \in P_{w}} C_{p}\left(x^{*}\right) \times\left[x_{p}-x_{p}^{*}\right] \geq 0, \quad \forall x \in \mathcal{K}^{5} .
$$

We now provide the standard variational inequality form of (23). In particular, we define the function that enters the variational inequality $F(x) \equiv C(x)$ and the feasible set $\mathcal{K} \equiv \mathcal{K}^{5}$. We then seek to determine $x^{*} \in \mathcal{K}$ such that

$$
\left\langle F\left(x^{*}\right), x-x^{*}\right\rangle \geq 0, \quad \forall x \in \mathcal{K},
$$

where $\langle\cdot, \cdot\rangle$ denotes the inner product in $n$-dimensional space where $n$ here is equal to the dimension of path flows, that is, $Q$. In Section 5, we present a dynamic version of the transportation network equilibrium problem formulated as an evolutionary variational inequality and we will use this standard form in connecting the static formulation with the dynamic one.

We now provide the equivalent variational inequality in link flows, which will be utilized in the demonstration of the supernetwork equivalence in Section 4. For additional background, see the book by Nagurney (1999) and the references therein. 


\section{Theorem 2}

A link flow pattern is a transportation network equilibrium if and only if it satisfies the variational inequality problem: determine $f^{*} \in \mathcal{K}^{6}$ satisfying

$$
\sum_{a \in L} c_{a}\left(f^{*}\right) \times\left(f_{a}-f_{a}^{*}\right) \geq 0, \quad \forall f \in \mathcal{K}^{6}
$$

where $\mathcal{K}^{6} \equiv\left\{f \in R_{+}^{K} \mid\right.$ there exists an $x$ satisfying $(18)$ and $\left.(19)\right\}$.

The continuity of the link cost functions and the compactness of the feasible sets $\mathcal{K}^{5}$ and $\mathcal{K}^{6}$ guarantee the existence of solutions to both variational inequalities (23) and (24) from the standard theory of variational inequalities (see Kinderlehrer and Stampacchia (1980)). 


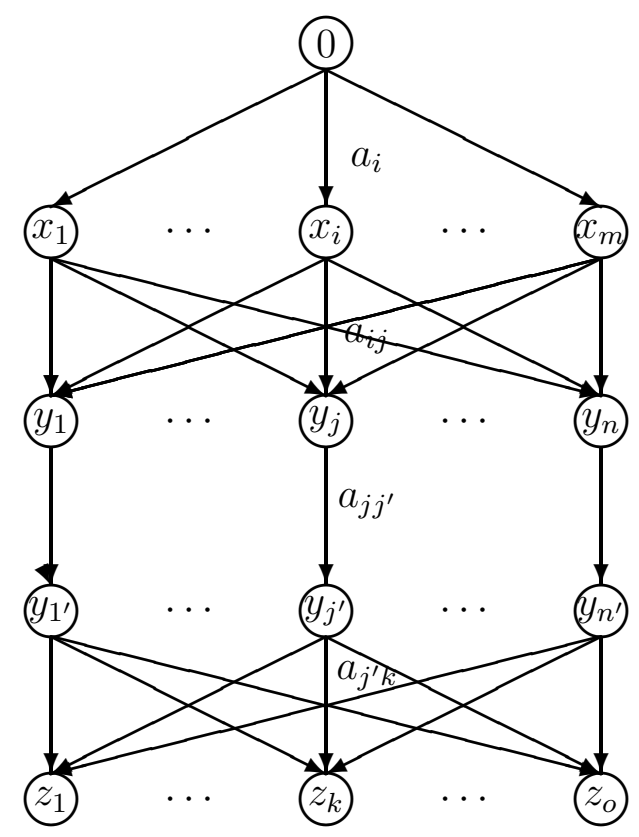

Figure 2: The $\mathcal{G}_{\mathcal{S}}$ Supernetwork Representation of Supply Chain Network Equilibrium with Fixed Demands

\section{Transportation Network Equilibrium Reformulation of Supply Chain Network Equilibrium with Fixed Demands}

In this Section, we establish the supernetwork equivalence of the fixed demand supply chain network equilibrium with a properly configured transportation network equilibrium model as discussed in Section 3.

We consider a supply chain network as discussed in Section 2 which consists of $m$ manufacturers: $i=1, \ldots, m$; $n$ retailers: $j=1, \ldots, n$, and $o$ demand markets: $k=1, \ldots, o$. The supernetwork $\mathcal{G}_{\mathcal{S}}$ of the isomorphic transportation network equilibrium model is depicted in Figure 2 and is constructed as follows. The supernetwork $\mathcal{G}_{\mathcal{S}}$ consists of the single origin node 0 at the top tier, and $o$ destination nodes at the bottom tier denoted, respectively, by: $z_{1}, \ldots, z_{o}$. Thus, there are $o \mathrm{O} / \mathrm{D}$ pairs in $\mathcal{G}_{\mathcal{S}}$ denoted, respectively, by $w_{1}=\left(0, z_{1}\right)$, $\ldots, w_{k}=\left(0, z_{k}\right), \ldots, w_{o}=\left(0, z_{o}\right)$. Node 0 is connected to each second-tiered node $x_{i}$, where $i=1, \ldots, m$. Each second-tiered node $x_{i}$, in turn, is connected to each third tiered node 
$y_{j}$, where $j=1, \ldots, n$. Each node $y_{j}$, in turn, is connected with a corresponding node $y_{j}^{\prime}$ in the fourth tier by a single link. Finally, from each fourth-tiered node $y_{j}^{\prime}$ there are $o$ links emanating to the bottom tiered nodes $z_{k}$. There are, hence, $1+m+2 n+o$ nodes in the supernetwork in Figure 2, $K=m+m n+n+n o$ links, $Z=o \mathrm{O} / \mathrm{D}$ pairs, and $Q=m o$ paths.

We now define the links in the supernetwork in Figure 2 and the associated flows. Let $a_{i}$ denote the link from node 0 to node $x_{i}$ with associated link flow $f_{a_{i}}$, for $i=1, \ldots, m$. Let $a_{i j}$ denote the link from node $x_{i}$ to node $y_{j}$ with associated link flow $f_{a_{i j}}$ for $i=1, \ldots, m$ and $j=1, \ldots, n$. Also, let $a_{j j^{\prime}}$ denote the link connecting node $y_{j}$ with node $y_{j^{\prime}}$ with associated link flow $f_{a_{j j^{\prime}}}$ for $j ; j=1, \ldots, n$; and $j^{\prime} ; j^{\prime}=1, \ldots, n$. Finally, let $a_{j^{\prime} k}$ denote the link joining node $y_{j^{\prime}}$ with node $z_{k}$ for $j^{\prime}=1^{\prime}, \ldots, n^{\prime}$ and $k=1, \ldots, o$ and with associated link flow $f_{a_{j^{\prime} k}}$. We group the $\left\{f_{a_{i}}\right\}$ into the vector $f^{1}$; the $\left\{f_{a_{i j}}\right\}$ into the vector $f^{2}$; the $\left\{f_{a_{j j^{\prime}}}\right\}$ into the vector $f^{3}$, and the $\left\{f_{a_{j^{\prime} k}}\right\}$ into the vector $f^{4}$.

Hence, a typical path in $\mathcal{G}_{\mathcal{S}}, p_{i j j^{\prime} k}$, consists of four links: $a_{i}, a_{i j}, a_{j j^{\prime}}$, and $a_{j^{\prime} k}$. We denote the path flow associated with path $p_{i j j^{\prime} k}$ by $x_{p_{i j j^{\prime} k}}$. Also, we let $d_{w_{k}}$ denote the known fixed demand associated with O/D pair $w_{k}$ and we let $\lambda_{w_{k}}$ denote the travel disutility associated with $\mathrm{O} / \mathrm{D}$ pair $w_{k}$.

We assume that the link flows satisfy the conservation of flow equations (19), that is:

$$
\begin{gathered}
f_{a_{i}}=\sum_{j=1}^{n} \sum_{j^{\prime}=1^{\prime}}^{n^{\prime}} \sum_{k=1}^{o} x_{p_{i j j^{\prime} k} k}, \quad i=1, \ldots, m, \\
f_{a_{i j}}=\sum_{j^{\prime}=1^{\prime}}^{n^{\prime}} \sum_{k=1}^{o} x_{p_{i j j^{\prime} k}}, \quad i=1, \ldots, m ; j=1, \ldots, n, \\
f_{a_{j j^{\prime}}}=\sum_{i=1}^{m} \sum_{k=1}^{o} x_{p_{i j j^{\prime} k}}, \quad j=1, \ldots, n ; j^{\prime}=1, \ldots, n, \\
f_{a_{j^{\prime} k}}=\sum_{i=1}^{m} \sum_{j=1}^{n} x_{p_{i j j^{\prime} k}}, \quad j^{\prime}=1, \ldots, n ; k=1, \ldots, o .
\end{gathered}
$$

Also, we have that

$$
d_{w_{k}}=\sum_{i=1}^{m} \sum_{j=1}^{n} \sum_{j^{\prime}=1^{\prime}}^{n^{\prime}} x_{i j j^{\prime} k}, \quad k=1, \ldots, o .
$$


A path flow pattern induces a feasible link flow pattern if all path flows are nonnegative and (25)-(29) are satisfied.

Given a feasible product shipment/transaction pattern for the supply chain model with fixed demands, $\left(q, Q^{1}, s, Q^{2}\right) \in \mathcal{K}^{4}$, we may construct a feasible link flow pattern on the network $\mathcal{G}_{\mathcal{S}}$ as follows: the link flows are defined as:

$$
\begin{gathered}
q_{i} \equiv f_{a_{i}}, \quad i=1, \ldots, m, \\
q_{i j} \equiv f_{a_{i j}}, \quad i=1, \ldots, m ; j=1, \ldots, n \\
s_{j} \equiv f_{a_{j j^{\prime}}}, \quad j=1, \ldots, n ; j^{\prime}=1, \ldots, n^{\prime}, \\
q_{j k}=f_{a_{j^{\prime} k}}, \quad j=1, \ldots, n ; j^{\prime}=1^{\prime}, \ldots, n^{\prime} ; k=1, \ldots, o .
\end{gathered}
$$

Note that if $\left(q, Q^{1}, s, Q^{2}\right)$ is feasible then the link flow pattern constructed according to (30) - (33) is also feasible and the corresponding path flow pattern that induces such a link flow pattern is, hence, also feasible.

We now assign travel costs on the links of the network $\mathcal{G}_{\mathcal{S}}$ as follows: with each link $a_{i}$ we assign a travel $\operatorname{cost} c_{a_{i}}$ defined by

$$
c_{a_{i}} \equiv \frac{\partial f_{i}}{\partial q_{i}}, \quad i=1, \ldots, m
$$

with each link $a_{i j}$ we assign a travel cost $c_{a_{i j}}$ defined by:

$$
c_{a_{i j}} \equiv \frac{\partial c_{i j}}{\partial q_{i j}}, \quad i=1, \ldots, m ; j=1, \ldots, n ;
$$

and with each link $j j^{\prime}$ we assign a travel cost defined by

$$
c_{a_{j j^{\prime}}} \equiv \frac{\partial c_{j}}{\partial s_{j}}, \quad j=1, \ldots, n ; j^{\prime}=1, \ldots, n
$$

Finally, for each link $a_{j^{\prime} k}$ we assign a travel cost defined by

$$
c_{a_{j^{\prime} k}} \equiv c_{j k}, \quad j^{\prime}=1, \ldots, n^{\prime} ; k=1, \ldots, o
$$


Hence, a traveler traveling on path $p_{i j j^{\prime} k}$ experiences a travel cost $C_{p_{i j j^{\prime} k}}$ given by

$$
C_{p_{i j j^{\prime} k}}=c_{a_{i}}+c_{a_{i j}}+c_{a_{j j^{\prime}}}+c_{a_{j^{\prime} k}}=\frac{\partial f_{i}}{\partial q_{i}}+\frac{\partial c_{i j}}{\partial q_{i j}}+\frac{\partial c_{j}}{\partial s_{j}}+c_{j k} .
$$

Also, we define the travel demands associated with the O/D pairs as follows:

$$
d_{w_{k}} \equiv d_{k}, \quad k=1, \ldots, o
$$

and the travel disutilities:

$$
\lambda_{w_{k}} \equiv \rho_{3 k}, \quad k=1, \ldots, o .
$$

Consequently, according to the fixed demand transportation network equilibrium conditions (22), we have that, for each O/D pair $w_{k}$ in $\mathcal{G}_{\mathcal{S}}$ and every path connecting the O/D pair $w_{k}$, the following conditions must hold:

$$
C_{p_{i j j^{\prime} k}}-\lambda_{w_{k}}^{*}=\frac{\partial f_{i}}{\partial q_{i}}+\frac{\partial c_{i j}}{\partial q_{i j}}+\frac{\partial c_{j}}{\partial q_{j}}+c_{j k}-\lambda_{w_{k}}^{*}\left\{\begin{array}{lll}
=0, & \text { if } & x_{p_{i j j^{\prime} k}}^{*}>0 \\
\geq 0, & \text { if } & x_{p_{i j j^{\prime} k}}^{*}=0
\end{array}\right.
$$

where

$$
\sum_{p \in P_{w_{k}}} x_{p_{i j j^{\prime} k}}^{*}=d_{w_{k}}
$$

We now provide the variational inequality formulation of the equilibrium conditions (41) in link form as in (24). A link flow pattern $f^{*} \in \mathcal{K}^{6}$ is an equilibrium according to (41), if and only if it satisfies:

$$
\begin{gathered}
\sum_{i=1}^{m} c_{a_{i}}\left(f^{1 *}\right) \times\left(f_{a_{i}}-f_{a_{i}}^{*}\right)+\sum_{i=1}^{m} \sum_{j=1}^{n} c_{a_{i j}}\left(f^{2 *}\right) \times\left(f_{a_{i j}}-f_{a_{i j}}^{*}\right) \\
+\sum_{j=1}^{n} \sum_{j^{\prime}=1^{\prime}}^{n^{\prime}} c_{a_{j j^{\prime}}}\left(f^{3 *}\right) \times\left(f_{a_{j^{\prime}}}-f_{a_{j j^{\prime}}}^{*}\right)+\sum_{j^{\prime}=1}^{n^{\prime}} \sum_{k=1}^{n} c_{a_{j^{\prime} k}}\left(f^{4 *}\right) \times\left(f_{a_{j^{\prime} k}}-f_{a_{j^{\prime} k}}^{*}\right) \geq 0, \quad \forall f \in \mathcal{K}^{6},
\end{gathered}
$$

which, through expressions (30) - (33), and (34) - (37) yields:

$$
\sum_{i=1}^{m}\left[\frac{\partial f_{i}\left(q^{*}\right)}{\partial q_{i}}\right] \times\left[q_{i}-q_{i}^{*}\right]+\sum_{i=1}^{m} \sum_{j=1}^{n}\left[\frac{\partial c_{i j}\left(q_{i j}^{*}\right)}{\partial q_{i j}}\right] \times\left[q_{i j}-q_{i j}^{*}\right]
$$




$$
+\sum_{j=1}^{n}\left[\frac{\partial c_{j}\left(s^{*}\right)}{\partial s_{j}}\right] \times\left[s_{j}-s_{j}^{*}\right]+\sum_{j=1}^{n} \sum_{k=1}^{o}\left[c_{j k}\left(Q^{2 *}\right)\right] \times\left[q_{j k}-q_{j k}^{*}\right] \geq 0, \quad \forall\left(q, Q^{1}, s, Q^{2}\right) \in \mathcal{K}^{4}
$$

But variational inequality (44) is precisely variational inequality (16) governing the supply chain network equilibrium with fixed demands.

Hence, we have the following result:

\section{Theorem 3}

A solution $\left(q^{*}, Q^{1 *}, s^{*}, Q^{2 *}\right) \in \mathcal{K}^{4}$ of the variational inequality (16) governing a supply chain network equilibrium coincides with the (via (30) - (33) and (34) - (37)) feasible link flow for the supernetwork $\mathcal{G}_{\mathcal{S}}$ constructed above and satisfies variational inequality (24); equivalently, variational inequality (43). Hence, it is a transportation network equilibrium according to Theorem 2.

We now describe how to recover the prices in the supply chain network with fixed demands. The vector of equilibrium prices $\rho_{3}^{*}$ associated with the product at the demand markets can be obtained by setting (cf. (40) and (41)): $\rho_{3 k}^{*}=C_{p_{i j j^{\prime} k}}=\lambda_{w_{k}}^{*}$ for each demand market $k$. The vector of equilibrium prices $\rho_{2}^{*}$ associated with retailers, in turn, can be obtained by setting (cf. (8) and (37)): $\rho_{2 j}^{*}=\lambda_{w_{k}}^{*}-c_{a_{j^{\prime} k}}=\left[\rho_{3 k}^{*}-c_{j k}\left(Q^{2 *}\right)\right]$ for any $j, k$ such that $q_{j k}^{*}>0$. The equilibrium prices $\rho_{1 i j}^{*}$, in turn, can be recovered by setting (cf. (3), (34), and $(35)): \rho_{1 i j}^{*}=c_{a_{i}}+c_{a_{i j}}=\left[\frac{\partial f_{i}\left(Q^{1 *}\right)}{\partial q_{i j}}+\frac{\partial c_{i j}\left(q_{i j}^{*}\right)}{\partial q_{i j}}\right]$ for any $i, j$ such that $q_{i j}^{*}>0$.

We now further discuss the interpretation of the supply chain network equilibrium conditions. These conditions define the supply chain network equilibrium in terms of paths and path flows, which coincide with Wardrop's (1952) first principle of user-optimization in the context of transportation networks over the network given in Figure 2. Thus, we have an entirely new interpretation of supply chain network equilibrium in the case of known demands, which is as follows: all used paths connecting the source node 0 and a particular destination node have equal and minimal costs, and the cost on the utilized paths for this O/D pair is equal to the disutility (or the demand market price) that the consumers pay.

It is worth noting that the above identification yields and yet another application that can be formulated and solved as a transportation network equilibrium problem. For additional 
applications, including spatial price equilibrium problems and Walrasian price equilibrium problems, see Nagurney (1999) and the references therein.

We also point out that for a relatively price-insensitive product, such as, for example, gasoline or milk, the fixed demand assumption is, indeed, practical, and we expect that the model will provide a good approximation. We further emphasize that the equivalence established above between supply chain networks and transportation networks with fixed demands provides new opportunities for further modeling enhancements. In Section 5, we exploit this equivalence when we develop a dynamic supply chain network equilibrium model with time-varying demand. 


\section{Dynamic Supply Chain Networks with Time-Varying Demands}

In this Section, we utilize the isomorphic transportation network established in Section 4 to develop a dynamic supply chain network model using an evolutionary variational inequality formulation. In Daniele, Maugeri, and Oettli (1998, 1999), evolutionary variational inequalities were utilized to model time-dependent transportation equilibria (see also Ran and Boyce (1996) and the references therein). Cojocaru, Daniele, and Nagurney (2005) demonstrated that time-dependent transportation equilibrium problems, as well as related dynamic spatial price equilibrium problems, and financial equilibrium problems, could be unified under a general evolutionary variational inequality definition over a unified constraint set. Nagurney, Liu, Cojocaru, and Daniele (2005) exploited the supernetwork equivalence between electric power networks and transportation networks, and developed an evolutionary variational inequality model for time-dependent electric power generation, distribution, and consumption. See the book by Nagurney (2006b) for additional theory and applications of dynamic supply chains.

In this Section, we consider the nonempty, convex, closed, bounded subset of the Hilbert space $L^{2}\left([0, T], R^{Q}\right)$ (where $T$ denotes the time interval under consideration and $\mu=$ constant and very large) given by

$$
\hat{\mathcal{K}}=\left\{x \in L^{2}\left([0, T], R^{Q}\right): 0 \leq x(t) \leq \mu \text { a.e. in }[0, T] ; \sum_{p \in P_{w}} x_{p}(t)=d_{w}(t), \forall w \text {, a.e. in }[0, T]\right\} .
$$

Hence, for definiteness, and greater ease in relating the discussion to the existing literature, we, without any loss of generality, consider the vector of path flows on the network at time $t$ to be denoted by $x(t)$ with an individual element by $x_{p}(t)$ and with $d_{w}(t)$ denoting the demand associated with $\mathrm{O} / \mathrm{D}$ pair $w$ at time $t$.

Thus, we assume that the demands, $d_{w}(t)$, for all O/D pairs $w$ are time-varying which means that the path flows will also change over time. We define:

$$
\langle\langle\Phi, x\rangle\rangle=\int_{0}^{T}\langle\Phi(t), x(t)\rangle d t
$$

where $\Phi \in L^{2}\left([0, T], R^{Q}\right)^{*}$ and $x \in L^{2}\left([0, T], R^{Q}\right)$. Let the function $F$ be $F: \hat{\mathcal{K}} \rightarrow$ 
$L^{2}\left([0, T], R^{Q}\right)$. We now provide the standardized form of the infinite-dimensional evolutionary (time-dependent) variational inequality (cf. Cojocaru, Daniele, and Nagurney (2005, 2006)): determine $x^{*} \in \hat{\mathcal{K}}$ such that:

$$
\left\langle\left\langle F\left(x^{*}\right), x-x^{*}\right\rangle\right\rangle \geq 0, \quad \forall x \in \hat{\mathcal{K}} .
$$

Sufficient conditions (including monotonicity-type conditions) that guarantee the existence of a solution to (47) are discussed in Daniele, Maugeri, and Oettli (1999).

Cojocaru, Daniele, and Nagurney (2006) have established that for the case of Hilbert spaces $H$ (namely, $L^{2}\left([0, T], R^{Q}\right)$ ) the following infinite-dimensional projected dynamical systems (PDS) can be related to the evolutionary variational inequality (EVI) (47) as follows:

$$
\frac{d x(t, \tau)}{d \tau}=\Pi_{\hat{\mathcal{K}}}(x(t, \tau),-F(x(t, \tau))), \quad x(t, 0) \in \hat{\mathcal{K}},
$$

where

$$
\Pi_{\hat{\mathcal{K}}}(y,-F(y))=\lim _{\delta \rightarrow 0^{+}} \frac{P_{\hat{\mathcal{K}}}((y-\delta F(y))-y)}{\delta}, \quad \forall y \in \hat{\mathcal{K}},
$$

with the projection operator $P_{\hat{\mathcal{K}}}: H \rightarrow \hat{\mathcal{K}}$ given by

$$
\left\|P_{\hat{\mathcal{K}}}(z)-z\right\|=\inf _{y \in \hat{\mathcal{K}}}\|y-z\| .
$$

Dupuis and Nagurney (1993) established the relationship between a projected dynamical system and a variational inequality in the case of finite dimensions. Cojocaru and Jonker (2004), subsequently, provided the relationship of the two formulations in infinitedimensional Hilbert spaces. Recently, Cojocaru, Daniele, and Nagurney (2006) showed the following:

\section{Theorem 4}

Assume that $\hat{\mathcal{K}} \subseteq H$ is non-empty, closed, and convex. Assume also that $F: \hat{\mathcal{K}} \rightarrow H$ is a pseudo-monotone vector field, that is, for every pair of points $x, y \in \hat{\mathcal{K}}$, we have that

$$
\langle F(x), y-x\rangle \Longrightarrow\langle F(y), y-x\rangle \geq 0,
$$


and that $F$ is Lipschitz continuous, where $H$ is a Hilbert space. Then the solutions of EVI (47) are the same as the critical points of the projected differential equation (48), that is, they are the functions $x^{*} \in \hat{\mathcal{K}}$ such that

$$
\Pi_{\hat{\mathcal{K}}}\left(x^{*}(t),-F\left(x^{*}(t)\right)\right)=0,
$$

and vice-versa.

Applying Theorem 4, we conclude that the solutions to the evolutionary variational inequality: determine $x^{*} \in \hat{\mathcal{K}}$ such that:

$$
\int_{0}^{T}\left\langle F\left(x^{*}(t)\right), x(t)-x^{*}(t)\right\rangle d t \geq 0, \quad \forall x \in \hat{\mathcal{K}},
$$

coincide with the critical points of the equation:

$$
\frac{d x(t, \tau)}{d \tau}=\Pi_{\hat{\mathcal{K}}}(x(t, \tau),-F(x(t, \tau))),
$$

that is, the points satisfying

$$
\Pi_{\hat{\mathcal{K}}}\left(x^{*}(t, \tau),-F\left(x^{*}(t, \tau)\right)\right) \equiv 0 \text { a.e. in }[0, T],
$$

which are apparently stationary with respect to $\tau$.

Note that in the formulation of the infinite-dimensional PDS (53), there are two "times," the meaning of which is discussed in Cojocaru, Daniele, and Nagurney (2007). Intuitively, at each moment $t \in[0, T]$, the solution of the evolutionary variational inequality (47) represents a static state of the underlying system. As $t$ varies over $[0, T]$, the static states describe one (or more) curves of the equilibria. On the other hand, $\tau$ here is the time that describes the dynamics of the system until it reaches one of the equilibria of the curve.

The dynamic, evolutionary variational inequality analogue of the static, finite-dimensional variational inequality (23) is now immediate. We substitute the vector of path costs into (47) and we obtain the evolutionary variational inequality for time-dependent transportation network equilibria given by: determine $x^{*} \in \hat{\mathcal{K}}$ such that:

$$
\left\langle\left\langle C\left(x^{*}\right), x-x^{*}\right\rangle\right\rangle \geq 0, \quad \forall x \in \hat{\mathcal{K}},
$$


where $C$ is the vector of path costs.

According to Theorem 3, the supply chain network equilibrium problem with fixed demands can be reformulated as a fixed demand transportation network equilibrium problem over the supernetwork $\mathcal{G}_{\mathcal{S}}$ given in Figure 2. Hence, the evolutionary variational inequality (55), in turn, provides us now with a dynamic version of the supply chain network model in which the demands vary over time, where the path costs are given by (38) and these are functions of path flows that now vary with time.

In the next Section, we illustrate the dynamic supply chain network model with concrete numerical examples. 


\section{Dynamic Numerical Supply Chain Network Examples with Computations}

In this Section, we provide numerical examples in order to demonstrate how the theoretical results in this paper can be applied in practice. In particular, we consider numerical supply chain network examples with time-varying demands and product flows.

To solve the associated evolutionary variational inequality, we utilize the approach set forth in Cojocaru, Daniele, and Nagurney $(2005,2006,2007)$, in which the time horizon $T$ is discretized and at each fixed point in time we solve the associated projected dynamical system (cf. also Nagurney and Zhang (1996)). We have chosen the examples so that the corresponding vector field $F$ satisfies the requirements in Theorem 4 (see also Nagurney, Dong, and Zhang (2002)), which we expect to be readily fulfilled in practice.

We utilized the Euler method for our numerical computations. The Euler method is induced by the general iterative scheme of Dupuis and Nagurney (1993) and has been applied by Nagurney and Zhang (1996) and Zhang and Nagurney (1997) to solve the variational inequality problem (23) in path flows as well as to approximate the continuous time trajectories associated with the corresponding projected dynamical system until the stationary point is attained. We applied the Euler method at discrete time points over the time interval $T$. Obviously, this procedure is correct if the continuity of the solution is guaranteed. Continuity results for solutions to evolutionary variational inequalities, in the case where $F(x(t))=A(t) x(t)+B(t)$ is a linear operator, $A(t)$ is a continuous and positive definite matrix in $[0, T]$, and $B(t)$ is a continuous vector can be found in Barbagallo (2007). In the examples that we present here such assumptions are fulfilled. Of course, the examples could also be computed via the computational procedure given in Daniele, Maugeri, and Oettli (1999) but here we utilize a time-discretization approach which also has intuitive appeal.

The Euler method was implemented in FORTRAN and the computer system used was a Sun system at the University of Massachusetts at Amherst. The convergence criterion utilized was that the absolute value of the path flows between two successive iterations differed by no more than $10^{-5}$. The sequence $\left\{\alpha_{\tau}\right\}$ in the Euler method (cf. Nagurney

and Zhang (1996)) was set to: .1\{1, $\left.\frac{1}{2}, \frac{1}{2}, \frac{1}{3}, \frac{1}{3}, \frac{1}{3}, \ldots\right\}$. The Euler method was initialized by distributing the demand for each $\mathrm{O} / \mathrm{D}$ pair equally among the paths connecting the respective O/D pair for each discretized point in time. 


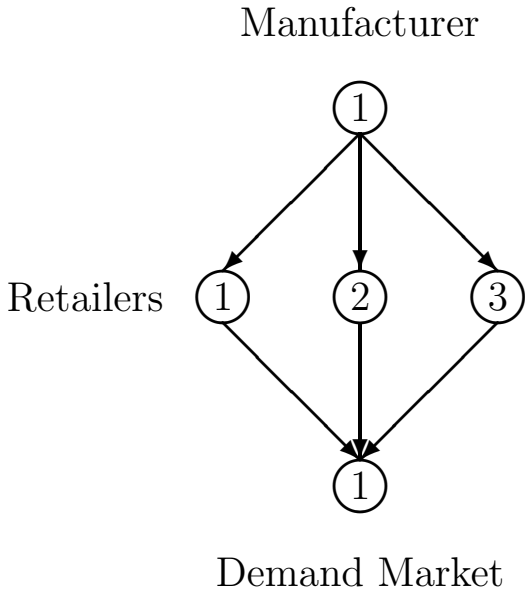

Demand Market

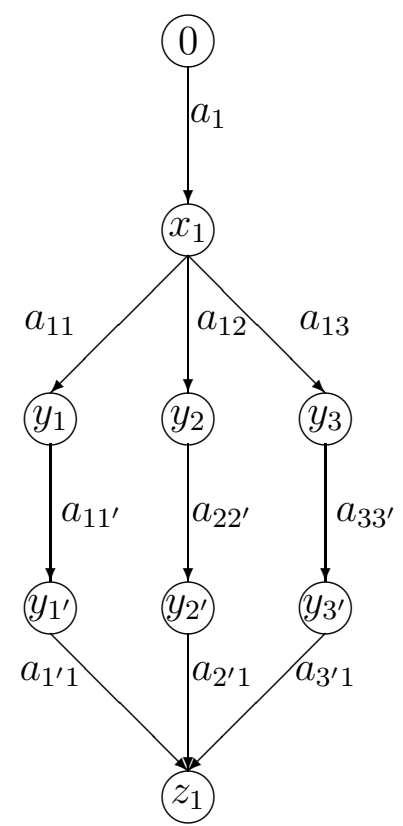

Corresponding Supernetwork

Figure 3: Supply Chain Network and Corresponding Supernetwork $\mathcal{G}_{\mathcal{S}}$ for Numerical Examples 1 and 2 


\section{Example 1}

In the first numerical example, the supply chain network consisted of one manufacturer, three retailers, and one demand market as depicted in Figure 3. The supernetwork representation which allows for the transformation (as proved in Section 4) to a transportation network equilibrium problem is given also in Figure 3. Hence, in the first numerical example (see also Figure 2) we had that: $m=1, n=3, n^{\prime}=3^{\prime}$, and $o=1$.

The notation is presented here in the form of the supply chain network model as delineated in Table 1. We provide the complete supernetwork representation in terms of $\mathrm{O} / \mathrm{D}$ pairs, paths, etc. The translations of the equilibrium path flows, link flows, and travel disutilities into the equilibrium flows and prices is then given, for completeness, and easy reference.

The production cost function for the manufacturer was given by:

$$
f_{1}(q(t))=2.5 q_{1}(t)^{2}+2 q_{1}(t) .
$$

The transaction cost functions faced by the manufacturer and associated with transacting with the retailers were given by:

$$
\begin{gathered}
c_{11}\left(q_{11}(t)\right)=.5 q_{11}(t)^{2}+3.5 q_{11}(t), \quad c_{12}\left(q_{12}(t)\right)=.5 q_{12}(t)^{2}+2.5 q_{12}(t), \\
c_{13}\left(q_{13}(t)\right)=.5 q_{13}(t)^{2}+1.5 q_{13}(t) .
\end{gathered}
$$

The operating costs of the retailers, in turn, were given by:

$$
c_{1}\left(Q^{1}(t)\right)=.5\left(q_{11}(t)\right)^{2}, \quad c_{2}\left(Q^{1}(t)\right)=.5\left(q_{12}(t)\right)^{2}, \quad c_{3}\left(Q^{1}(t)\right)=.5\left(q_{13}(t)\right)^{2} .
$$

The unit transaction costs associated with transacting between the retailers and the demand market were:

$$
c_{11}\left(Q^{2}(t)\right)=q_{11}(t)+1, \quad c_{21}\left(Q^{2}(t)\right)=q_{21}(t)+5, \quad c_{31}\left(Q^{2}(t)\right)=q_{31}(t)+10 .
$$

We utilized the supernetwork representation of this example depicted in Figure 3 with the links enumerated as in Figure 3 in order to solve the problem via the Euler method. Note 
that there are 9 nodes and 10 links in the supernetwork in Figure 3. Using the procedure outlined in Section 4 , we defined O/D pair $w_{1}=\left(0, z_{1}\right)$ with the user link travel cost functions as given in $(34)-(37)$.

There were three paths in $P_{w_{1}}$ denoted by: $p_{1}, p_{2}, p_{3}$. The paths were comprised of the following links:

$$
p_{1}=\left(a_{1}, a_{11}, a_{11^{\prime}}, a_{1^{\prime} 1}\right), \quad p_{2}=\left(a_{1}, a_{12}, a_{22^{\prime}}, a_{2^{\prime} 1}\right), \quad p_{3}=\left(a_{1}, a_{13}, a_{33^{\prime}}, a_{3^{\prime} 1}\right)
$$

The time horizon $T=1$. The time-varying demand function was given by:

$$
d_{1}(t)=100+10 t
$$

We discretized the time horizon $T$ as follows: $t_{0}=0, t_{1}=\frac{1}{2}$, and $t_{2}=T=1$. We report the solutions obtained by the Euler method at each discrete time step, for which we had, respectively, demands: $d_{1}\left(t_{0}\right)=100 ; d_{1}\left(t_{1}\right)=105$, and $d_{1}(T)=110$.

Example 1: Solution at Time $t=t_{0}=0$ :

The Euler method converged and yielded the following equilibrium path flow pattern:

$$
x_{p_{1}}^{*}\left(t_{0}\right)=34.44, \quad x_{p_{2}}^{*}\left(t_{0}\right)=33.44, \quad x_{p_{3}}^{*}\left(t_{0}\right)=32.12 .
$$

The corresponding equilibrium link flows (cf. also the supernetwork in Figure 3) were:

$$
\begin{gathered}
f_{a_{1}}^{*}\left(t_{0}\right)=100.00, \\
f_{a_{11}}^{*}\left(t_{0}\right)=34.44, \quad f_{a_{12}}^{*}\left(t_{0}\right)=33.44, \quad f_{a_{13}}^{*}\left(t_{0}\right)=32.12, \\
f_{a_{11^{\prime}}}^{*}\left(t_{0}\right)=34.44, \quad f_{a_{22^{\prime}}}^{*}\left(t_{0}\right)=33.44, \quad f_{a_{33^{\prime}}}^{*}\left(t_{0}\right)=32.12, \\
f_{a_{1^{\prime} 1}}^{*}\left(t_{0}\right)=34.44, \quad f_{a_{2^{\prime}}}^{*}\left(t_{0}\right)=33.44, \quad f_{a_{3^{\prime}}}^{*}\left(t_{0}\right)=32.12 .
\end{gathered}
$$

The incurred equilibrium path travel costs (cf. (38)) were: $C_{p_{1}}\left(t_{0}\right)=C_{p_{2}}\left(t_{0}\right)=C_{p_{3}}\left(t_{0}\right)=$ $\lambda_{w_{1}}^{*}\left(t_{0}\right)=609.83$ 
We now provide the translations of the above equilibrium flows into the supply chain network flow and price notation using (30), (31), (32), and (33).

The flows were:

$$
\begin{gathered}
Q^{1 *}\left(t_{0}\right):=q_{11}^{*}\left(t_{0}\right)=34.44, \quad q_{12}^{*}\left(t_{0}\right)=33.44, \quad q_{13}^{*}\left(t_{0}\right)=32.12, \\
s_{1}^{*}\left(t_{0}\right)=34.44, \quad s_{2}^{*}\left(t_{0}\right)=33.44, \quad s_{3}^{*}\left(t_{0}\right)=32.12, \\
Q^{2 *}\left(t_{0}\right):=q_{11}^{*}\left(t_{0}\right)=34.44, \quad q_{21}^{*}\left(t_{0}\right)=33.44, \quad q_{31}^{*}\left(t_{0}\right)=32.12,
\end{gathered}
$$

and the production quantity was: $q_{1}^{*}\left(t_{0}\right)=100$.

The demand price at the demand market was, hence, (cf. (40)):

$$
\rho_{31}^{*}\left(t_{0}\right)=609.83
$$

which corresponds to the travel costs on the paths (all are used) connecting the O/D pair.

It is easy to verify that the equilibrium conditions were satisfied with excellent accuracy.

Example 1: Solution at Time $t=t_{1}=\frac{1}{2}$ :

The Euler method converged and yielded the following equilibrium path flow pattern:

$$
x_{p_{1}}^{*}\left(t_{1}\right)=36.11, \quad x_{p_{2}}^{*}\left(t_{1}\right)=35.11, \quad x_{p_{3}}^{*}\left(t_{1}\right)=33.78 .
$$

The corresponding equilibrium link flows (cf. also the supernetwork in Figure 3) were:

$$
\begin{gathered}
f_{a_{1}}^{*}\left(t_{1}\right)=105.00, \\
f_{a_{11}}^{*}\left(t_{1}\right)=36.11, \quad f_{a_{12}}^{*}\left(t_{1}\right)=35.11, \quad f_{a_{13}}^{*}\left(t_{1}\right)=33.78, \\
f_{a_{1^{\prime}}}^{*}\left(t_{1}\right)=36.11, \quad f_{a_{22^{\prime}}}^{*}\left(t_{1}\right)=35.11, \quad f_{a_{33^{\prime}}}^{*}\left(t_{1}\right)=33.78, \\
f_{a_{1^{\prime}}}^{*}\left(t_{1}\right)=36.11, \quad f_{a_{2^{\prime}}}^{*}\left(t_{1}\right)=35.11, \quad f_{a_{3^{\prime}}}^{*}\left(t_{1}\right)=33.78,
\end{gathered}
$$

with a production quantity: $q_{1}^{*}\left(t_{1}\right)=105$. The equilibrium path travel costs were now: $C_{p_{1}}\left(t_{1}\right)=C_{p_{2}}\left(t_{1}\right)=C_{p_{3}}\left(t_{1}\right)=\lambda_{w_{1}}^{*}\left(t_{1}\right)=639.83$. 
The translations into the corresponding equilibrium supply chain flows at time $t_{1}$ can easily be done as described for time $t_{0}$.

The demand price at the demand market was now:

$$
\rho_{31}^{*}\left(t_{1}\right)=639.83
$$

which corresponds to the travel costs on the paths (all paths are again used) connecting the O/D pair.

It is easy to verify that the equilibrium conditions were again satisfied with excellent accuracy.

Example 1: Solution at Time $t=T=1$ :

We applied the Euler method to the end of the time horizon where $T=1$. The Euler method now yielded the following equilibrium path flow pattern:

$$
x_{p_{1}}^{*}(T)=37.77, \quad x_{p_{2}}^{*}(T)=36.77, \quad x_{p_{3}}^{*}(T)=35.45 .
$$

The corresponding equilibrium link flows (cf. also the supernetwork in Figure 3) were:

$$
\begin{aligned}
f_{a_{1}}^{*}(T)=110.00, \\
f_{a_{11}}^{*}(T)=37.77, \quad f_{a_{12}}^{*}(T)=36.77, \quad f_{a_{13}}^{*}(T)=35.45, \\
f_{a_{1^{\prime}}}^{*}(T)=37.77, \quad f_{a_{22^{\prime}}}^{*}(T)=36.77, \quad f_{a_{33^{\prime}}}^{*}(T)=35.45, \\
f_{a_{1^{\prime}}}^{*}(T)=37.77, \quad f_{a_{2^{\prime} 1}}^{*}(T)=36.77, \quad f_{a_{3^{\prime} 1}}^{*}(T)=35.45 .
\end{aligned}
$$

The translations into the corresponding equilibrium supply chain flows can be easily done as above for time $t_{0}$.

The demand price at the demand market was now:

$$
\rho_{31}^{*}(T)=669.83
$$

which is equal to $\lambda_{w_{1}}^{*}(T)=C_{p_{1}}(T)=C_{p_{2}}(T)=C_{p_{3}}(T)$. 


\section{Explicit Formulae for Example 1 for the Time-Dependent Equilibria}

We now note that, due to the linearity of $F$ in this example, as well as the separability of the components of $F$, and the special structure of the topology of the supernetwork in Figure 3 , we can write down explicit formulae for the path flows over time $[0, T]$. See also, Dafermos and Sparrow (1969) who made the same observation in the context of transportation network equilibrium problems on networks in which all paths connecting an O/D pair consisted of single links, and the user link cost functions were linear and separable. Cojocaru, Daniele, and Nagurney (2005, 2006) provided explicit formulae for solutions to dynamic transportation network examples of such special topologies and cost structures.

In particular, we obtain the following formulae for the equilibrium path flows for Example 1 at each point $t$ :

$$
\begin{aligned}
& x_{p_{1}}^{*}(t)=3.33 t+34.44, \\
& x_{p_{2}}^{*}(t)=3.33 t+33.44, \\
& x_{p_{3}}^{*}(t)=3.33 t+32.12,
\end{aligned}
$$

and these formulae are valid even for $T>1$, that is, outside the range [0,1], which is of concern here. We also have an explicit formula for the travel disutility where:

$$
\lambda_{w_{1}}^{*}(t)=60 t+609.83, \quad \text { for } \quad t \in[0, T]
$$

We now, for completeness, translate these formulae into supply chain network model formulae (30) - (33) with time-varying flows (see also (55)). Please refer also to the supernetwork in Figure 3. In particular, we have the time-dependent equilibrium supply chain flows are given by:

$$
\begin{gathered}
q_{1}^{*}(t)=f_{a_{1}}^{*}(t)=x_{p_{1}}^{*}(t)+x_{p_{2}}^{*}(t)+x_{p_{3}}^{*}(t)=10 t+100 ; \\
Q^{1 *}(t):=q_{11}^{*}(t)=f_{a_{11}}^{*}(t)=x_{p_{1}}^{*}(t)=3.33 t+34.33, \quad q_{12}^{*}(t)=f_{a_{12}}^{*}(t)=x_{p_{2}}^{*}(t)=3.33 t+33.44, \\
q_{13}^{*}(t)=f_{a_{13}}^{*}(t)=x_{p_{3}}^{*}(t)=3.33 t+32.12 ; \\
s_{1}^{*}(t)=f_{a_{11^{\prime}}}^{*}(t)=x_{p_{1}}^{*}(t)=3.33 t+34.44, \quad s_{2}^{*}(t)=f_{22^{\prime}}^{*}(t)=x_{p_{2}}^{*}(t)=3.33 t+33.44, \\
s_{3}^{*}(t)=f_{a_{33^{\prime}}}^{*}(t)=x_{p_{3}}^{*}(t)=3.33 t+32.12,
\end{gathered}
$$


and

$$
\begin{gathered}
Q^{2 *}:=q_{11}^{*}(t)=f_{a_{1^{\prime} 1}}^{*}(t)=x_{p_{1}}^{*}(t)=3.33 t+34.44, \quad q_{21}^{*}(t)=f_{a_{2^{\prime} 1}}^{*}(t)=x_{p_{2}}^{*}(t)=3.33 t+33.44 \\
q_{31}^{*}(t)=f_{a_{3^{\prime} 1}}^{*}(t)=x_{p_{3}}^{*}(t)=3.33 t+32.12 .
\end{gathered}
$$

\section{Example 2: A Numerical Supply Chain Example with Step-wise Time Varying Demand}

The second example had the same data as Example 1, except that the demand now had a step-wise structure. The supply chain network and the supernetwork were, hence, as in Figure 3. In particular, the demand was of the form given below on the time interval $[0, T]$ :

$$
d_{1}(t)= \begin{cases}s_{1}, & \text { if } \quad 0<t \leq t_{1}, \\ s_{2}, & \text { if } \quad t_{1}<t \leq t_{2}, \\ \ldots, & \end{cases}
$$

where, in this example, we have that:

$$
d_{1}(t)= \begin{cases}100, & \text { if } \quad 0<t \leq t_{1}=\frac{1}{2} \\ 110, & \text { if } \quad t_{1}<t \leq t_{2}=T=1\end{cases}
$$

Such a structure may reflect, for example, a seasonable demand for a product

In this setting, we know that the equilibrium curve (solution of the evolutionary variational inequality) is a step function, with the steps given by the function $d_{1}(t)$, where:

$$
x^{*}(t)= \begin{cases}x_{1}^{*}, & \text { if } \quad 0<t \leq t_{1}=\frac{1}{2} \\ x_{2}^{*}, & \text { if } \quad t_{1}<t \leq t_{2}=1=T .\end{cases}
$$

Again, given the simplicity of the supernetwork topology and the cost structure, we obtain an explicit solution:

$$
x^{*}(t)=\left(x_{p_{1}}^{*}(t), x_{p_{2}}^{*}(t), x_{p_{3}}^{*}(t)\right)= \begin{cases}(34.44,33.44,32.12), & \text { if } \quad 0<t \leq t_{1}=\frac{1}{2}, \\ (37.77,36.77,35.45), & \text { if } \quad t_{1}<t \leq t_{2}=1=T .\end{cases}
$$

Of course, the transformation of the these equilibrium path flows into the equilibrium link flows and the supply chain network flows can be done as was done for Example 1 since the supernetwork topology is one and the same for Examples 1 and 2. 


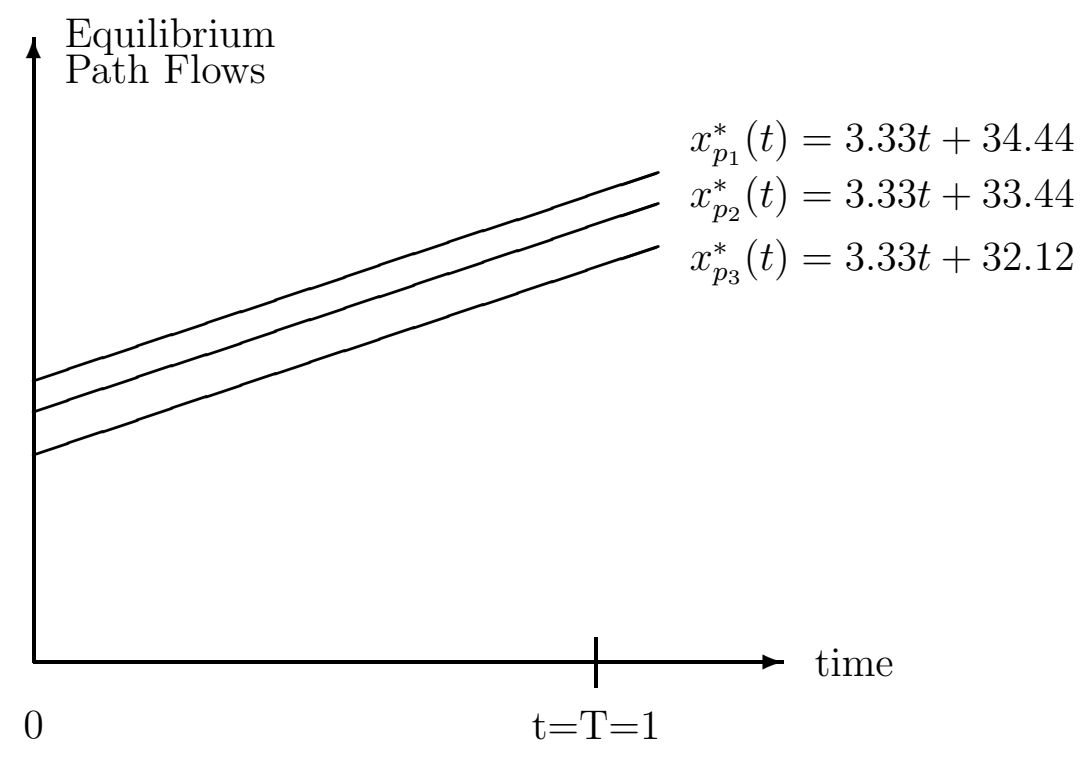

Figure 4: Time-Dependent Equilibrium Path Flows for Example 1

In Figures 4 and 5 we provide the graphs of the time-dependent equilibrium path flows for Examples 1 and 2, respectively. 


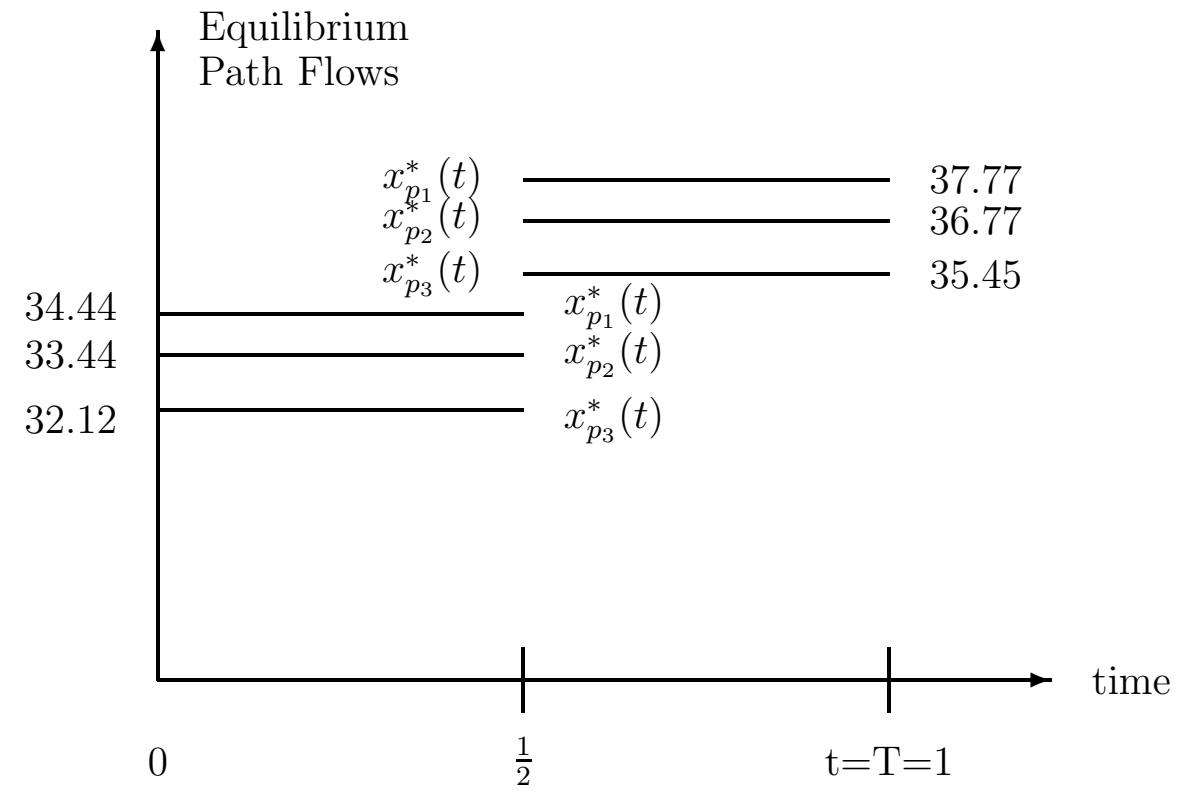

Figure 5: Time-Dependent Equilibrium Path Flows for Example 2 with Step-Wise Demand 


\section{Example 3}

In the third numerical example, the supply chain network consisted of two manufacturers, one retailer, and two demand markets. Hence, we now had that $m=2, n=1, n^{\prime}=1^{\prime}$, and $o=2$.

The data were now as follows: The production cost functions for the manufacturers were given by:

$$
f_{1}(q(t))=2.5 q_{1}(t)^{2}+q_{1}(t) q_{2}(t)+2 q_{1}(t), \quad f_{2}(q(t))=2.5 q_{2}(t)^{2}+q_{2}(t) q_{1}(t)+2 q_{2}(t) .
$$

The transaction cost functions faced by the manufacturers and associated with transacting with the retailers were given by:

$$
c_{11}\left(q_{11}(t)\right)=.5 q_{11}(t)^{2}+3.5 q_{11}(t), \quad c_{21}\left(q_{21}(t)\right)=.5 q_{21}(t)^{2}+1.5 q_{21}(t) .
$$

The operating cost of the retailer, in turn, was given by:

$$
c_{1}\left(Q^{1}(t)\right)=.5\left(q_{11}(t)\right)^{2}
$$

The unit transaction costs associated with transacting between the retailers and the demand market were:

$$
c_{j k}\left(Q^{2}(t)\right)=q_{j k}(t)+1, \quad \text { for } j=1,2 ; k=1,2 .
$$

We utilized the supernetwork representation of this example depicted in Figure 6 with the links enumerated as in Figure 6 in order to solve the problem via the Euler method. Note that there are 7 nodes and 7 links in the supernetwork in Figure 6. Using the procedure outlined in Section 4 , we defined O/D pair $w_{1}=\left(0, z_{1}\right)$ and O/D pair $w_{2}=\left(0, z_{2}\right)$ with the user link travel cost functions as given in (40) - (43).

There were two paths in $P_{w_{1}}$ denoted by: $p_{1}, p_{2}$ and two paths in $P_{w_{2}}$ denoted by: $p_{3}$ and $p_{4}$, respectively. The paths were comprised of the following links:

$p_{1}=\left(a_{1}, a_{11}, a_{11^{\prime}}, a_{1^{\prime} 1}\right), p_{2}=\left(a_{2}, a_{21}, a_{11^{\prime}}, a_{1^{\prime} 1}\right), p_{3}=\left(a_{1}, a_{11}, a_{11^{\prime}}, a_{1^{\prime} 2}\right), p_{4}=\left(a_{2}, a_{21}, a_{11^{\prime}}, a_{1^{\prime} 2}\right)$. 


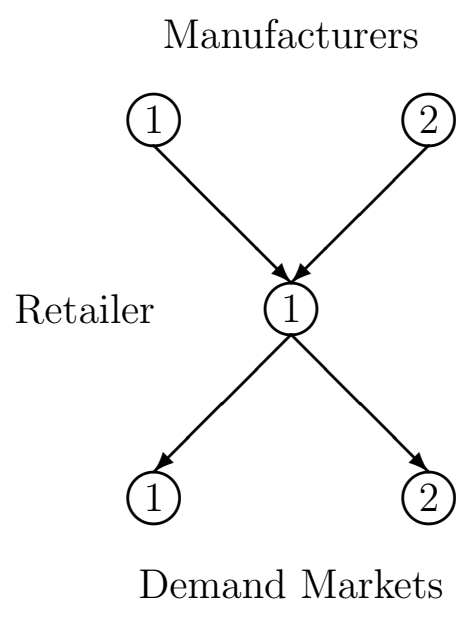

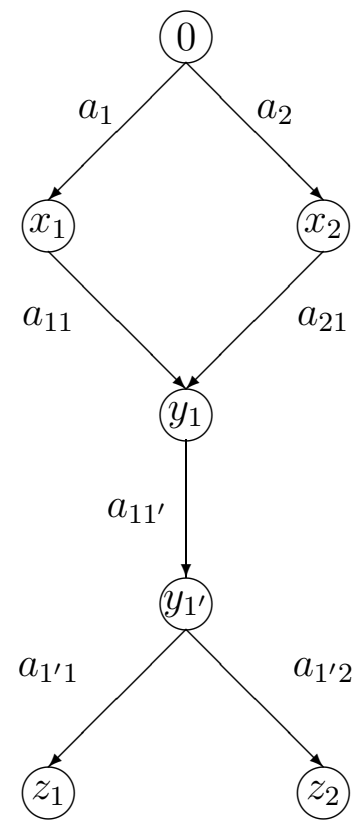

Corresponding Supernetwork

Figure 6: Supply Chain Network and Corresponding Supernetwork $\mathcal{G}_{\mathcal{S}}$ for Numerical Example 3 
The time horizon $T=1$. The time-varying demand functions were given by:

$$
d_{1}(t)=100+5 t, \quad d_{2}(t)=80+4 t .
$$

We discretized the time horizon $T$ as follows: $t_{0}=0, t_{1}=\frac{1}{2}$, and $t_{2}=T=1$. We report the solutions obtained by the Euler method at each discrete time step, for which we had, respectively, demands: $d_{1}\left(t_{0}\right)=100, d_{1}\left(t_{1}\right)=102.5$, and $d_{1}(T)=105$, and $d_{2}\left(t_{0}\right)=80$, $d_{2}\left(t_{1}\right)=82$, and $d_{2}(T)=84$.

Example 3: Solution at Time $t=t_{0}=0$ :

We applied the Euler method to the beginning of the time horizon where $t=t_{0}=0$. The Euler method now yielded the following equilibrium path flow pattern:

$$
x_{p_{1}}^{*}\left(t_{0}\right)=49.90, \quad x_{p_{2}}^{*}\left(t_{0}\right)=50.10, \quad x_{p_{3}}^{*}\left(t_{0}\right)=39.90, \quad x_{p_{4}}^{*}\left(t_{0}\right)=40.10 .
$$

The corresponding equilibrium link flows (cf. also the supernetwork in Figure 4) were:

$$
\begin{gathered}
f_{a_{1}}^{*}\left(t_{0}\right)=89.80, \quad f_{a_{2}}^{*}\left(t_{0}\right)=90.20 \\
f_{a_{11}}^{*}\left(t_{0}\right)=89.80, \quad f_{a_{21}}^{*}\left(t_{0}\right)=90.20, \\
f_{a_{11^{\prime}}}^{*}\left(t_{0}\right)=180.00, \\
f_{a_{1^{\prime} 1}}^{*}\left(t_{0}\right)=100.00, \quad f_{a_{1^{\prime}}}^{*}\left(t_{0}\right)=80.00,
\end{gathered}
$$

with incurred equilibrium path travel costs: $C_{p_{1}}\left(t_{0}\right)=C_{p_{2}}\left(t_{0}\right)=\lambda_{w_{1}}^{*}\left(t_{0}\right)=815.50$ and $C_{p_{3}}\left(t_{0}\right)=C_{p_{4}}\left(t_{0}\right)=\lambda_{w_{2}}^{*}\left(t_{0}\right)=815.50$.

The translations into the corresponding equilibrium flows are now given:

$$
\begin{aligned}
Q^{1 *}\left(t_{0}\right):=q_{11}^{*}\left(t_{0}\right)=89.80, \quad q_{21}^{*}\left(t_{0}\right)=92.90, \\
s_{1}^{*}\left(t_{0}\right)=f_{a_{11^{\prime}}}^{*}\left(t_{0}\right)=180.00, \\
Q^{2 *}\left(t_{0}\right):=q_{11}^{*}\left(t_{0}\right)=100.00, \quad q_{12}^{*}\left(t_{0}\right)=80.00 .
\end{aligned}
$$


The demand prices at the demand markets were:

$$
\rho_{31}^{*}\left(t_{0}\right)=815.50, \quad \rho_{32}^{*}\left(t_{0}\right)=815.50
$$

which correspond to the travel costs on the paths (all are used) connecting the respective O/D pair.

Example 3: Solution at Time $t=t_{1}=\frac{1}{2}$ :

We applied the Euler method to time $t=t_{1}=\frac{1}{2}$. The Euler method now yielded the following equilibrium path flow pattern:

$$
x_{p_{1}}^{*}\left(t_{1}\right)=51.15, \quad x_{p_{2}}^{*}\left(t_{1}\right)=51.35, \quad x_{p_{3}}^{*}\left(t_{1}\right)=40.90, \quad x_{p_{4}}^{*}\left(t_{1}\right)=41.10 .
$$

The corresponding equilibrium link flows (cf. also the supernetwork in Figure 3) were:

$$
\begin{gathered}
f_{a_{1}}^{*}\left(t_{1}\right)=92.05, \quad f_{a_{2}}^{*}\left(t_{1}\right)=92.45 \\
f_{a_{11}}^{*}\left(t_{1}\right)=92.05, \quad f_{a_{21}}^{*}\left(t_{1}\right)=92.45, \\
f_{a_{11^{\prime}}}^{*}\left(t_{1}\right)=184.50, \\
f_{a_{1^{\prime} 1}}^{*}\left(t_{1}\right)=102.50, \quad f_{a_{1^{\prime} 2}}^{*}\left(t_{1}\right)=82.00,
\end{gathered}
$$

with equilibrium path costs:

$$
C_{p_{1}}\left(t_{1}\right)=C_{p_{2}}\left(t_{1}\right)=\lambda_{w_{1}}^{*}\left(t_{1}\right)=835.75, \quad C_{p_{3}}\left(t_{1}\right)=C_{p_{4}}\left(t_{1}\right)=\lambda_{w_{2}}^{*}\left(t_{1}\right)=835.75 .
$$

The translations into the corresponding equilibrium supply chain flows are now given:

$$
\begin{gathered}
Q^{1 *}\left(t_{1}\right):=q_{11}^{*}\left(t_{1}\right)=92.05, \quad q_{21}^{*}\left(t_{1}\right)=92.45, \\
s_{1}^{*}\left(t_{1}\right)=f_{a_{11^{\prime}}}^{*}\left(t_{1}\right)=184.50, \\
Q^{2 *}\left(t_{1}\right):=q_{11}^{*}\left(t_{1}\right)=102.50, \quad q_{12}^{*}\left(t_{1}\right)=82.00 .
\end{gathered}
$$

The demand prices at the demand markets were now:

$$
\rho_{31}^{*}\left(t_{1}\right)=835.75, \quad \rho_{32}^{*}\left(t_{1}\right)=835.75
$$


which correspond to the travel costs on the paths (all are used) connecting the respective O/D pair.

\section{Example 3: Solution at Time $t=T=1$ :}

Finally, we applied the Euler method to the end of the time horizon where $t=T$. The Euler method now yielded the following equilibrium path flow pattern:

$$
x_{p_{1}}^{*}(T)=52.40, \quad x_{p_{2}}^{*}(T)=52.60, \quad x_{p_{3}}^{*}(T)=41.90, \quad x_{p_{4}}^{*}(T)=42.10 .
$$

The corresponding equilibrium link flows (cf. Figure 4) were:

$$
\begin{array}{r}
f_{a_{1}}^{*}(T)=94.30, \quad f_{a_{2}}^{*}(T)=94.70, \\
f_{a_{11}}^{*}(T)=94.30, \quad f_{a_{21}}^{*}(T)=94.70, \\
f_{a_{11^{\prime}}}^{*}(T)=189.00, \\
f_{a_{1^{\prime} 1}}^{*}(T)=105.00, \quad f_{a_{1^{\prime}}}^{*}(T)=84.00 .
\end{array}
$$

The equilibrium path costs, in turn, were now:

$$
C_{p_{1}}(T)=C_{p_{2}}(T)=\lambda_{w_{1}}^{*}(T)=856.00, \quad C_{p_{3}}(T)=C_{p_{4}}(T)=\lambda_{w_{2}}^{*}(T)=856.00 .
$$

The translations into the corresponding equilibrium supply chain flows were, hence:

$$
\begin{aligned}
Q^{1 *}(T):= & q_{11}^{*}(T)=94.30, \quad q_{21}^{*}(T)=94.70, \\
& s_{1}^{*}(T)=f_{a_{11^{\prime}}}^{*}(T)=189.00, \\
Q^{2 *}(T):= & q_{11}^{*}(T)=105.00, \quad q_{12}^{*}(T)=84.00 .
\end{aligned}
$$

The demand prices at the demand markets were now:

$$
\rho_{31}^{*}(T)=856.00, \quad \rho_{32}^{*}(T)=856.00
$$

which correspond to the travel costs on the paths (all are used) connecting the respective O/D pair. 


\section{References}

Barbagallo, A., 2007. Regularity results for time-dependent variational and quasi-variational inequalities and application to the calculation of dynamic traffic network. Mathematical Models and Methods in Applied Sciences 17, no. 3, 1-28.

Bazaraa, M. S., Sherali, H. D., Shetty, C. M., 1993. Nonlinear Programming: Theory and Algorithms. John Wiley \& Sons, New York.

Beckmann, M. J., McGuire, C. B., Winsten, C. B., 1956. Studies in the Economics of Transportation. Yale University Press, New Haven, Connecticut.

Boyce, D. E., Mahmassani, H. S., Nagurney, A., 2005. A retrospective on Beckmann, McGuire, and Winsten's Studies in the Economics of Transportation. Papers in Regional Science 84, 85-103.

Cojocaru, M.-G., Daniele, P., Nagurney, A., 2005. Projected dynamical systems and evolutionary variational inequalities via Hilbert spaces with applications. Journal of Optimization Theory and Applications 27, no. 3, 1-15.

Cojocaru, M.-G., Daniele, P., Nagurney, A., 2006. Double-layered dynamics: A unified theory of projected dynamical systems and evolutionary variational inequalities. European Journal of Operational Research 175, 494-507.

Cojocaru, M.-G., Daniele, P., Nagurney, A., 2007. Projected dynamical systems, evolutionary variational inequalities, applications, and a computational procedure. Pareto Optimality, Game Theory and Equilibria. A. Migdalas, P. M. Pardalos, and L. Pitsoulis, editors, Springer Verlag, in press.

Cojocaru, M.-G., Jonker, L. B., 2004. Existence of solutions to projected differential equations in Hilbert spaces. Proceedings of the American Mathematical Society 132, 183-193.

Cournot, A. A., 1838. Researches into the Mathematical Principles of the Theory of Wealth. English translation, MacMillan, England. 
Dafermos, S., 1980. Traffic equilibrium and variational inequalities. Transportation Science $14,42-54$.

Dafermos, S., 1982. The general multimodal network equilibrium problem with elastic demand. Networks 12, 57-72.

Dafermos, S., Nagurney, A., 1984. Stability and sensitivity analysis for the general network equilibrium - travel choice model. In: Volmuller, J., Hamerslag, R. (Eds.), Proceedings of the Ninth International Symposium on Transportation and Traffic Theory. VNU Science Press, Utrecht, The Netherlands, pp. 217-232.

Dafermos, S., Nagurney, A., 1987. Oligopolistic and competitive behavior of spatially separated markets. Regional Science and Urban Economics 17, 245-254.

Dafermos, S. C., Sparrow, F. T., 1969. The traffic assignment problem for a general network. Journal of Research of the National Bureau of Standards 73B, 91-118.

Daniele, P., Maugeri, A., Oettli, W., 1998. Variational inequalities and time-dependent traffic equilibria. Comptes Rendue Academie des Science, Paris 326, serie I, 10591062.

Daniele, P., Maugeri, A., Oettli, W., 1999. Time-dependent traffic equilibria. Journal of Optimization Theory and its Applications 103, 543-555.

Dupuis, P., Nagurney, A., 1993. Dynamical systems and variational inequalities. Annals of Operations Research 44, 9-42.

Fisk, C., Boyce, D. E., 1983. Alternative variational inequality formulation of the network equilibrium-travel choice problem. Transportation Science 17, 454-463.

Gabay, D., Moulin, H., 1980. On the uniqueness and stability of Nash equilibria in noncooperative games. In: Applied Stochastic Control in Econometrics and Management Science, Bensoussan, A., Kleindorfer, P., Tapiero, C. S. (Eds.), North-Holland, Amsterdam, The Netherlands, 271-294.

Kinderlehrer, D., Stampacchia, G., 1980. An Introduction to Variational Inequalities and 
Their Applications. Academic Press, New York.

Nagurney, A., 1999. Network Economics: A Variational Inequality Approach, second and revised edition. Kluwer Academic Publishers, Dordrecht, The Netherlands.

Nagurney, A. 2006. On the relationship between supply chain and transportation network equilibria: A supernetwork equivalence with computations. Transportation Research E 42, 293-316 .

Nagurney, A. 2006b. Supply Chain Network Economics: Dynamics of Prices, Flows, and Profits, Edward Elgar Publishing, Cheltenham, England.

Nagurney, A., Dong, J., 2002. Supernetworks: Decision-Making for the Information Age. Edward Elgar Publishing, Cheltenham, England.

Nagurney, A., Dong, J., Zhang, D., 2002. A supply chain network equilibrium model. Transportation Research E 38, 281-303.

Nagurney, A., Liu, Z., Cojocaru, M., Daniele, P., 2005. Static and dynamic transportation network equilibrium reformulations of electric power supply chain networks with known demands, to appear in Transportation Research E.

Nagurney, A., Zhang, D., 1996. Projected Dynamical Systems and Variational Inequalities with Applications. Kluwer Academic Publishers, Boston, Massachusetts.

Nagurney, A., Zhang, D., 1997. Projected dynamical systems in the formulation, stability analysis, and computation of fixed demand traffic network equilibria. Transportation Science $31,147-158$.

Nash, J. F., 1950. Equilibrium points in n-person games. Proceedings of the National Academy of Sciences 36, 48-49.

Nash, J. F., 1951. Noncooperative games. Annals of Mathematics 54, 286-298.

Ran, B., Boyce, D. E., 1996. Modeling Dynamic Transportation Networks. Springer-Verlag, Berlin, Garmany. 
Smith, M. J., 1979. Existence, uniqueness, and stability of traffic equilibria. Transportation Research 13B, 259-304.

Wardrop, J. G., 1952. Some theoretical aspects of road traffic research. In: Proceedings of the Institution of Civil Engineers, Part II 1, 325-378.

Zhang, D., Nagurney, A., 1997. Formulation, stability, and computation of traffic network equilibria as projected dynamical systems. Journal of Optimization Theory and Applications 93, 417-444.

Zhang, P., Peeta, S., Friesz, T. L., 2005. Dynamic game theoretic model of multi-layer infrastructure networks. Networks and Spatial Economics 5, 147-178. 